\title{
Real-Gas Effects on Mean Flow and Temporal Stability of Binary-Species Mixing Layers
}

\author{
Nora Okong'o* and Josette Bellan ${ }^{\dagger}$ \\ Jet Propulsion Laboratory, California Institute of Technology, Pasadena, California 91109-8099
}

\begin{abstract}
Real-gas effects on the mean flow and inviscid stability of temporal mixing layers are examined for supercritical heptane/nitrogen and oxygen/hydrogen mixtures. The analysis is based on the compressible Navier-Stokes equations for conservation of mass, momentum, total energy, and species mass, with heat and species-mass fluxes derived from fluctuation-dissipation theory and incorporating Soret and Dufour effects. An approximate form of the equations is used to obtain a system of similarity equations for the streamwise velocity, the temperature, and the mass fraction. The similarity profiles show important real-gas nonideal-mixture effects, particularly for the temperature, in departing from the incompressible error-function similarity solution. Realistic Schmidt and Prandtl numbers were found to be important to the similarity profiles. A linear, inviscid stability analysis is then performed using the similarity profile, as well as analytical error-function profiles, as its basic flow. The stability analysis shows that the similarity profile has larger growth rates at a given wavelength and a shorter more unstable wavelength than the error-function profiles and than an incompressible flow. The similarity profile also has a larger range of unstable wavelengths than the error-function profiles.
\end{abstract}

\section{Introduction}

$\mathbf{S}$ UPERCRITICAL fluids are of great interest in extraction processes as well as in propulsiondevices such as liquid rockets and advanced gas-turbine and diesel engines. We here define a binary fluid mixture to be in a supercritical state when at a thermodynamic pressure $p$ or temperature $T$ exceeding the critical (subscript $c$ ) value $\left(p_{c}\right.$ or $T_{c}$ ) (Ref. 1). This definition is consistent with that for a single-species fluid, where in the supercritical state only one phase can exist. ${ }^{2}$ Sometimes the supercritical state is defined to be in the region where both the pressure and temperature are above their critical values; ${ }^{3}$ however, there are many subtleties to the definition of the supercritical region. For a single-species fluid, $p_{c}$ and $T_{c}$ are the maximum pressure and temperature at which two phases (liquid and vapor) may exist, ${ }^{1,2}$ and therefore, the single-phase region is well delineated $\left(p_{r} \equiv p / p_{c}>1\right.$ or $\left.T_{r} \equiv T / T_{c}>1\right)$. The possible complications of retrograde condensation near the critical point ${ }^{1,2}$ are not taken into account in this characterization. However, a binary mixture may have one or several critical loci, as shown by Prausnitz et al., ${ }^{1}$ who presented at least six types or classes of binary mixtures, with five having multiple critical loci. To avoid the confusion that may be introduced for such mixtures having several critical loci, these types of mixtures are not considered; instead the focus is on simple mixtures, that is, those having a single critical locus. The supercritical region is here defined as that for which only a single phase is possible and one in which the transport properties are generally pressure as well as temperature dependent. Because the criterion of $p_{r}>1$ or $T_{r}>1$ represents the thermodynamic region for which only a single phase is possible, it corresponds to our definition of the supercritical region. This definition is consistent with the utilization in the present study of a Peng-Robinson-type real-gas equation of state. In the practical situations motivating the present study, fluid enters a chamber that is pressurized above the critical pressure of the injected fluid. The injected fluid then mixes in a highly turbulent manner with the chamber fluid and disintegrates

Received 19 February 2003; revision received 10 July 2003; accepted for publication 27 August 2003. Copyright (C) 2003 by the California Institute of Technology. Published by the American Institute of Aeronautics and Astronautics, Inc., with permission. Copies of this paper may be made for personal or internal use, on condition that the copier pay the $\$ 10.00$ per-copy fee to the Copyright Clearance Center, Inc., 222 Rosewood Drive, Danvers, MA 01923; include the code 0001-1452/03 \$10.00 in correspondence with the CCC

* Scientist, 4800 Oak Grove Drive. Member AIAA.

Senior Research Scientist, 4800 Oak Grove Drive; josette.bellan@ jpl.nasa.gov. Associate Fellow AIAA. into smaller parcels, which participate in subsequent ignition and combustion. Clearly, fluid disintegration and turbulent mixing play a crucial role in determining the size and composition of the parcels of fluid and, consequently, the efficiency of combustion.

Many aspects of the fluid dynamics of interest can be examined by considering the turbulent mixing layer. Generally, the mixing layer is the region between two fluid streams of different velocity, temperature, and composition. For binary mixing layers, each stream contains a pure species; for incompressible flows, typically only the fluid stream velocitiesdiffer. A useful methodology to study turbulent flows is direct numerical simulation (DNS) in which all relevant scales are resolved. The high computational demands of DNS presently limit it to transitional Reynolds numbers $R e$ in geometrically simple configurations. One such configuration is the temporal mixing layer, which embodies the shear dynamics essential to turbulence, while simultaneously benefiting from the simplicity of periodic boundaries in the streamwise and spanwise directions. The temporal mixing layer is in important ways akin to the physically realizable spatial mixing layer, yet it allows simulations on a much smaller physical domain. It is roughly equivalent to following a small number of coherent structures as they grow in time, but does not exhibit the stream asymmetry of the spatial mixing layer. For temporal mixing layer simulations, judicious specification of the initial conditions, in particular, the imposition of perturbations at specified wavelengths on the given mean flow, may accelerate the attainment of a transitional state.

Moser and Rogers ${ }^{4}$ have done extensive work on incompressible temporal mixing layers, particularly investigating the form and amplitude of spanwise and streamwise perturbations. In their work, the wavelength used for the spanwise perturbations was the most unstable wavelength from a linear inviscid stability analysis based on an error function mean velocity profile, which is the similarity solution for the viscous incompressiblelayer. Metcalfe et al..$^{5}$ have performed similar simulations, but used hyperbolictangent mean profiles, with perturbations at the most unstable wavelength. For incompressible flow, Rayleigh's inflection point theorem (see Drazin and Reid ${ }^{6}$ ) shows that a necessary condition for inviscid instability is that the velocity profile has at least one inflection point. Both the erf and the tanh profiles satisfy this condition. The justification for using the most unstable wavelength in turbulent flow simulations is that it has been observed to dominate in many experimental situations, that is, spatial mixing layers, and that in the temporal mixing layer it would have the fastest growth rate, hence, reach the transitional $R e$ values fastest. Furthermore, at the $R e$ values under consideration, the development of the large structures will be governed by inviscid 
Table 1 Pure species properties

\begin{tabular}{lrrcc}
\hline \hline Species & $m, \mathrm{~g} / \mathrm{mol}$ & $T_{c}, \mathrm{~K}$ & $p_{c}, \mathrm{MPa}$ & $v_{c},{ }^{\mathrm{a}} \mathrm{cm}^{3} / \mathrm{mol}$ \\
\hline $\mathrm{H}_{2}$ & 2.016 & 33.0 & 1.284 & 64.3 \\
$\mathrm{~N}_{2}$ & 28.013 & 126.3 & 3.399 & 89.8 \\
$\mathrm{O}_{2}$ & 31.999 & 154.6 & 5.043 & 73.4 \\
$\mathrm{C}_{7} \mathrm{H}_{16}$ & 100.205 & 540.2 & 2.74 & 432.0 \\
\hline \hline
\end{tabular}

${ }^{a}$ Molar volume at the critical point.

instabilities. Therefore, a possible application of similarity and stability analyses is in determining mean flow and unstable excitation wavelengths, respectively, for DNS of mixing layers. However, a more fundamental relevance of similarity and stability analyses is in illuminating the effects of various flow properties by studying them in a simplified context, without turbulence.

In the realm of compressible flows, linear inviscid spatial stability analyses have been performed by Jackson and Grosch, ${ }^{7,8}$ Shin and Ferziger, ${ }^{9}$ Lu and Lele, ${ }^{10}$ and Kozusko et al. ${ }^{11}$ These analyses have shown that the growth rates are sensitive to freestream factors such as density ratio, species combination, and Mach number, as well as to the mean profile. Jackson and Grosch showed multiple modes for reacting flows ${ }^{7}$ and quantified the effect of various mean flow profiles ${ }^{8}$ (specifically tanh, Lock, and Sutherland models) on the stability characteristics, thereby formally justifying the use of the tanh profile instead of the similarity profile (although they did not use the erf profile in their studies). Shin and Ferziger ${ }^{9}$ derived necessary conditions for instability and showed that, based on the mean temperature profile for reacting flow, more than one instability mode may exist. The results of Kozusko et al. ${ }^{11}$ showed that a significant effect from the specification of the transport properties (viscosity, thermal conductivity, diffusivity) can occur; this effect enters through the mean profiles, which are based on viscous similarity solutions, because the stability analysis itself is inviscid and does not involve these properties.

The compressibleinvestigations listed were all devoted to perfect gases (PG) and ideal mixtures. However, our interest is in real-gas nonideal (RGNI) mixtures, such as those found in liquid rockets and gas-turbine and diesel engines. For high-pressure conditions, a real-gas equation of state (EOS) is essential to describe the mixture thermodynamics, as is the enlarged transport matrix containing Soret and Dufour effects. Moreover, consideration must be given to the possibly large density stratification for binary mixing layers at high pressures, and to the Schmidt and Prandtl number dependence on the thermodynamic state. In this paper, we present similarity solutions and linear stability analyses of RGNI temporal mixing layers. We first display the conservation equations suitable for three-dimensional simulations of RGNI temporal mixing layers and then derive approximate equations to compute similarity solutions. These similarity solutions are compared to the erf profile, and both profile types are used as mean flow profiles in the stability analysis. From linear inviscid stability analyses, we determine the unstable compressible wavelengths, which are compared with the incompressiblemost unstable wavelengths. All results presented herein are for supercritical heptane/nitrogen and oxygen/hydrogen mixtures; the critical properties of these species are presented in Table 1.

\section{Governing Equations}

The governing equations for the situation of interest have previously been published for heptane/nitrogen (Refs. 12-14) and oxygen/hydrogen (Ref. 15), where they were used for DNS of temporal transitional mixing layers. They are briefly summarized here to establish the context for the similarity and stability analyses.

\section{A. Conservation Equations}

The conservationequations originate from Keizer's ${ }^{16}$ fluctuationdissipation (FD) theory, which is consistent with nonequilibrium thermodynamics, converges to kinetic theory in the low-pressure limit, and relates fluxes and forces from first principles. For a non- reacting mixture, the conservation equations are

$$
\begin{gathered}
\frac{\partial \rho}{\partial t}+\frac{\partial\left(\rho u_{j}\right)}{\partial x_{j}}=0 \\
\frac{\partial\left(\rho u_{i}\right)}{\partial t}+\frac{\partial\left(\rho u_{i} u_{j}+p \delta_{i j}\right)}{\partial x_{j}}=\frac{\partial \tau_{i j}}{\partial x_{j}} \\
\frac{\partial\left(\rho Y_{\alpha}\right)}{\partial t}+\frac{\partial\left(\rho Y_{\alpha} u_{j}\right)}{\partial x_{j}}=-\frac{\partial j_{\alpha j}}{\partial x_{j}}, \quad \alpha=1, N \\
\frac{\partial\left(\rho e_{t}\right)}{\partial t}+\frac{\partial\left[\left(\rho e_{t}+p\right) u_{j}\right]}{\partial x_{j}}=-\frac{\partial q_{\mathrm{IK} j}}{\partial x_{j}}+\frac{\partial \tau_{i j} u_{i}}{\partial x_{j}}
\end{gathered}
$$

where $x$ is a Cartesian coordinate, $t$ is the time, $\rho$ is the density, $u_{i}$ is the $i$ th component of the velocity, $e_{t}=e+u_{i} u_{i} / 2$ is the total energy, that is, internal energy $e$ plus kinetic energy, $Y_{\alpha}$ is the mass fraction of species $\alpha$, and $\boldsymbol{j}_{\alpha}$ is its mass flux vector

$$
\sum_{\alpha=1}^{N} Y_{\alpha}=1, \quad \sum_{\alpha=1}^{N} j_{\alpha j}=0
$$

for a mixture of $N$ species. In the binary $(N=2)$ mixing layer configuration, the lighter (molar weight) species (subscript 1) will be in the upper stream, and the other species (subscript 2) will be in the lower stream. (See Table 1 for the molar weights.) Furthermore, $\boldsymbol{q}_{\mathrm{IK}}$ is the Irwing-Kirkwood (IK) form of the heat flux vector (see Sarman and Evans ${ }^{17}$ ), and $\tau_{i j}$ is the Newtonian viscous stress tensor

$$
\tau_{i j}=\mu\left(\frac{\partial u_{i}}{\partial x_{j}}+\frac{\partial u_{j}}{\partial x_{i}}-\frac{2}{3} \frac{\partial u_{k}}{\partial x_{k}} \delta_{i j}\right)
$$

where $\delta_{i j}$ is the Kronecker delta function, and $\mu$ is the mixture viscosity that is in general a function of the thermodynamic state variables. The mass flux and heat flux are given by

$$
\begin{gathered}
j_{2 j}=-\left[j_{2 j}^{\prime}+\left(\alpha_{\mathrm{IK}}-\alpha_{h}\right) Y_{2} Y_{1} \frac{\rho D}{T} \frac{\partial T}{\partial x_{j}}\right] \\
q_{\mathrm{IK} j}=-\lambda_{\mathrm{IK}}^{\prime} \frac{\partial T}{\partial x_{j}}-\alpha_{\mathrm{IK}} R_{u} T \frac{m}{m_{2} m_{1}} j_{2 j}^{\prime} \\
j_{2 j}^{\prime}=\rho D\left[\alpha_{D} \frac{\partial Y_{2}}{\partial x_{j}}+\frac{Y_{2} Y_{1}}{R_{u} T} \frac{m_{2} m_{1}}{m}\left(\frac{v_{, 2}}{m_{2}}-\frac{v_{, 1}}{m_{1}}\right) \frac{\partial p}{\partial x_{j}}\right] \\
\alpha_{h}=\frac{1}{R_{u} T} \frac{m_{2} m_{1}}{m}\left(\frac{h_{, 2}}{m_{2}}-\frac{h_{, 1}}{m_{1}}\right)
\end{gathered}
$$

The notation in Eqs. (6-9) is as follows: $D$ is the binary diffusion coefficient, $\alpha_{D}$ is the mass diffusion factor, $m_{\alpha}$ is the molar mass of species $\alpha$,

$$
m=\sum_{\alpha=1}^{N} m_{\alpha} X_{\alpha}
$$

is the mixture molar mass where the molar fraction $X_{\alpha}=m Y_{\alpha} / m_{\alpha}$, $v_{, \alpha}=\left(\partial v / \partial X_{\alpha}\right)_{T, p, X_{\beta}(\beta \neq \alpha)}$ is the partial molar volume, $h_{, \alpha}=$ $\left(\partial h / \partial X_{\alpha}\right)_{T, p, X_{\beta}(\beta \neq \alpha)}$ is the partial molar enthalpy,

$$
v=\sum_{\alpha=1}^{N} X_{\alpha} v_{, \alpha}
$$

is the molar volume related to the density by $v=m / \rho$,

$$
h=\sum_{\alpha=1}^{N} X_{\alpha} h_{, \alpha}
$$


Table 2 Transport properties for binary mixtures

\begin{tabular}{lcl}
\hline \hline & \multicolumn{1}{c}{ System } \\
\cline { 2 - 3 } Property $^{\mathrm{a}}$ & $\mathrm{C}_{7} \mathrm{H}_{16} / \mathrm{N}_{2}$ & \multicolumn{1}{c}{$\mathrm{O}_{2} / \mathrm{H}_{2}$} \\
\hline$Y_{2}$ & $\mathrm{C}_{7} \mathrm{H}_{16}$ mass fraction & $\mathrm{O}_{2}$ mass fraction \\
$\mu=\mu_{R}\left(T / T_{R}\right)^{n}$ & $n=0.7$ & $n=0.75$ \\
$S c \equiv \mu /\left(\rho \alpha_{D} D\right)$ & $1.5-Y_{2}$ & $\left(1.334-0.668 Y_{2}-0.186 Y_{2}^{2}-0.268 Y_{2}^{6}\right)\left[1+(88.6 / T)^{1.5}\right]$ \\
$P r \equiv \mu C_{p} /(m \lambda)$ & $0.5 S c / \exp \left(-1.5 Y_{2}\right)$ & $1.335 / T^{0.1}$ \\
$\alpha_{\mathrm{IK}}$ or $\alpha_{\mathrm{BK}}$ & $\alpha_{\mathrm{IK}}=0.1($ Ref. 18$)$ & $\alpha_{\mathrm{BK}}=0.2$ (Refs. 20 and 21$)$ \\
$T$ range, $\mathrm{K}$ & $500-1100$ & $200-800$ \\
$p$ range, atm & $40-80$ & $\sim 100$ \\
\hline \hline
\end{tabular}

${ }^{a}$ Reference temperature $T_{R}=\left(T_{1}+T_{2}\right) / 2, T$ in Kelvin.

is the molar enthalpy, $R_{u}$ is the universal gas constant, and $\lambda_{\mathrm{IK}}^{\prime}$ is a thermal conductivity defined from the transport matrix through

$$
\lambda_{\text {IK }}^{\prime}=\lambda+X_{1} X_{2} \alpha_{\mathrm{IK}} \alpha_{\mathrm{BK}} R_{u} \rho D / m
$$

with $\lim _{p \rightarrow 0} \lambda=\lambda_{\mathrm{KT}}$ as discussed by Harstad and Bellan, ${ }^{18}$ where KT refers to kinetic theory. The transport coefficients associated with the Soret (in the molar fluxes) and the Dufour (in the heat flux) terms of the transport matrix are $\alpha_{\mathrm{BK}}$ and $\alpha_{\mathrm{IK}}$, which are the two forms of the thermal diffusion factor corresponding to the IK and the Bearman-Kirkwood (BK) forms of the heat flux (see Sarman and Evans ${ }^{17}$ ). These transport coefficients are characteristic of each particular species pairs, and they obey the relationship ${ }^{18}$

$$
\alpha_{\mathrm{BK}}=\alpha_{\mathrm{IK}}-\alpha_{h}
$$

Additionally, $\lim _{p \rightarrow 0} \alpha_{\mathrm{IK}} \neq \alpha_{\mathrm{KT}}$ and $\lim _{p \rightarrow 0} \alpha_{\mathrm{BK}}=\alpha_{\mathrm{KT}}$.

\section{B. Equation of State}

The pressure is calculated from the well-known Peng-Robinson (PR) EOS, given $T$ and the PR molar volume $v_{\mathrm{PR}}$, as

$$
p=\frac{R_{u} T}{\left(v_{\mathrm{PR}}-b_{m}\right)}-\frac{a_{m}}{\left(v_{\mathrm{PR}}^{2}+2 b_{m} v_{\mathrm{PR}}-b_{m}^{2}\right)}
$$

where $a_{m}$ and $b_{m}$ are functions of $T$ and $X_{\alpha}$ (details in Ref. 12). At high pressures, $v_{\mathrm{PR}}$ may differ significantly from the actual molar volume $v$ (Ref. 1). Both $v_{\mathrm{PR}}$ and the volume shift $v_{S}=v-v_{\mathrm{PR}}$ can be calculated from the PR EOS given $p, T$, and $X_{\alpha}$ (Ref. 19), although for the $\mathrm{C}_{7} \mathrm{H}_{16} / \mathrm{N}_{2}$ system $v_{S}$ is negligible. All of the thermodynamic properties of interest are calculated in a consistent manner from the same $\operatorname{EOS}^{12,14,15}$; these properties are the molar enthalpy $h$, the constant pressure molar heat capacity $C_{p}=(\partial h / \partial T)_{p, X}$, and the speed of sound $a_{s}=1 / \sqrt{ }\left(\rho \kappa_{s}\right)$. The isentropic compressibility $\kappa_{s}$ is related to the expansivity $\alpha_{v}=(1 / v)(\partial v / \partial T)_{p, X}$, and the isothermal compressibility $\kappa_{T}=-(1 / v)(\partial v / \partial p)_{T, X}$ through $\kappa_{s}=\kappa_{T}-v T \alpha_{v}^{2} / C_{p}$. The mass diffusion factor $\alpha_{D}$ is calculated from the fugacity coefficients $\varphi_{\alpha}$ (which are related to the Gibbs energy) using $\alpha_{D}=1+X_{\alpha}\left[\partial\left(\ln \varphi_{\alpha}\right) / \partial X_{\alpha}\right]$ and portrays departures from mixture ideality, that is, $\alpha_{D}=1$.

\section{Transport Coefficients}

For the heptane/nitrogen and oxygen/hydrogen mixtures to be studied, the viscosity, the Schmidt number $S c$, and the Prandtl number $\operatorname{Pr}$, were calculated from high-pressure single-speciestransport properties using mixing rules, as by Harstad and Bellan. ${ }^{20}$ The calculated values were correlated, as summarized in Table 2, and these correlationswere then used to compute the transportproperties $\mu, D$, and $\lambda$. The thermal diffusion factors in Table 2 are from Refs. 18, 20, and 21 . The temperatures $T_{1}$ (upper, $\mathrm{H}_{2}$ or $\mathrm{N}_{2}$, stream) and $T_{2}$ (lower, $\mathrm{O}_{2}$ or $\mathrm{C}_{7} \mathrm{H}_{16}$, stream) correspond to the freestream temperatures for mixing layer simulations. Typically, for DNS, the value of the reference viscosity $\mu_{R}$ is determined by the specified value of the initial Reynolds number $R e_{0}$ [see Eq. (14)]. One of the thermal diffusion factors is specified, and then the other is calculated from Eq. (11).

\section{Configuration and Boundary Conditions}

The temporally developing mixing layer configuration is given in Fig. 1 for $\mathrm{C}_{7} \mathrm{H}_{16} / \mathrm{N}_{2}$, as an example, showing the definition of the streamwise, $x_{1}$, cross-stream, $x_{2}$, and spanwise, $x_{3}$, coordinates.

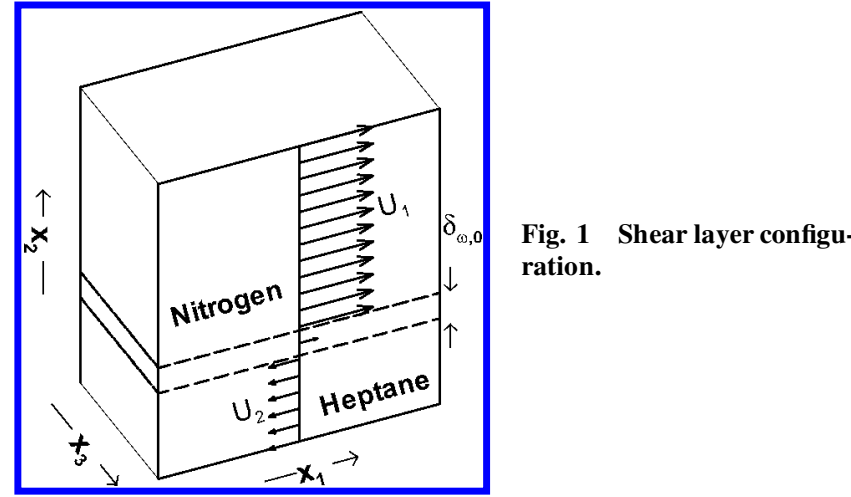

In the DNS of Refs. 14 and 15, the layer was not symmetric in extent in the $x_{2}$ direction, to accommodate the larger layer growth in the lighter fluid $\left(\mathrm{H}_{2}\right.$ or $\left.\mathrm{N}_{2}\right)$ side. The freestream density $\rho_{1}$ or $\rho_{2}$ is calculated for each pure species at its freestream temperature $T_{1}$ or $T_{2}$ and at the initial uniform pressure $p_{0}$. The vorticity thickness is defined as $\delta_{\omega}(t)=\Delta U_{0} /\left(\partial \bar{u}_{1} / \partial x_{2}\right)_{\max }$, where $\bar{u}_{1}$ is the $\left(x_{1}, x_{3}\right)$ planar average of the streamwise velocity and $\Delta U_{0}=U_{1}-U_{2}$ is the velocity difference across the layer.

For the present study, the freestream velocity is calculated using the relations of Papamoschou and Roshko, ${ }^{22}$

$$
U_{1}=2 M_{c, 0} a_{s_{1}}\left[1+\left(a_{s_{1}} / a_{s_{2}}\right) \sqrt{\rho_{1} / \rho_{2}}\right]^{-1}, \quad U_{2}=-\sqrt{\rho_{1} / \rho_{2}} U_{1}
$$

which lead to a convective velocity $U_{c}$ close to zero (Appendix A). Here $M_{c, 0}$ is the convective Mach number, whose specification, therefore, determines $\Delta U_{0}$. Given the initial streamwise velocity profile $u_{1}$ based on $U_{1}$ and $U_{2},\left(\partial \bar{u}_{1} / \partial x_{2}\right)_{\max }$ and, hence, $\delta_{\omega, 0} \equiv \delta_{\omega}(0)$ are calculated; $\mu_{R}$ is calculated from the specified value of $R e_{0}$,

$$
\operatorname{Re}_{0}=0.5\left(\rho_{1}+\rho_{2}\right) \Delta U_{0} \delta_{\omega, 0} / \mu_{R}
$$

In the temporal mixing layer configuration, periodic boundary conditions are used for the streamwise and spanwise directions, and nonreflecting outflow conditions are used in the cross-stream direction. ${ }^{13}$ The choice of $U_{c} \simeq 0$ results in the largest scale vortical structures being approximately stationary in the computational domain. ${ }^{15}$

\section{E. Primitive Form of Conservation Equations}

Some of the analysis to be presented will make use of the primitive form of Eqs. (1-4) for $N=2$ :

$$
\begin{aligned}
& \frac{\partial \rho}{\partial t}+\rho \frac{\partial u_{j}}{\partial x_{j}}+u_{j} \frac{\partial \rho}{\partial x_{j}}=0 \\
& \frac{\partial u_{i}}{\partial t}+u_{j} \frac{\partial u_{i}}{\partial x_{j}}+\frac{1}{\rho} \frac{\partial p}{\partial x_{i}}=\frac{1}{\rho} \frac{\partial \tau_{i j}}{\partial x_{j}} \\
& \frac{\partial Y_{2}}{\partial t}+u_{j} \frac{\partial Y_{2}}{\partial x_{j}}=-\frac{1}{\rho} \frac{\partial j_{2 j}}{\partial x_{j}}
\end{aligned}
$$




$$
\begin{aligned}
\frac{\partial T}{\partial t}+ & u_{j} \frac{\partial T}{\partial x_{j}}+\left(\frac{\kappa_{T}}{\kappa_{s}}-1\right) \frac{1}{\alpha_{v}} \frac{\partial u_{j}}{\partial x_{j}} \\
& =\frac{m}{\rho C_{p}} \frac{\kappa_{T}}{\kappa_{s}} \sum_{\alpha=1}^{2}\left[\frac{h_{\alpha}}{m_{\alpha}}+\left(\frac{\kappa_{s}}{\kappa_{T}}-1\right) \frac{C_{p}}{m \alpha_{v}}\left(\rho \frac{v_{\alpha}}{m_{\alpha}}\right)\right] \frac{\partial j_{\alpha j}}{\partial x_{j}} \\
& +\frac{m}{\rho C_{p}} \frac{\kappa_{T}}{\kappa_{s}}\left[-\frac{\partial q_{\mathrm{IK} j}}{\partial x_{j}}+\tau_{i j} \frac{\partial u_{i}}{\partial x_{j}}\right] \\
\frac{\partial p}{\partial t}+ & u_{j} \frac{\partial p}{\partial x_{j}}+\frac{1}{\kappa_{s}} \frac{\partial u_{j}}{\partial x_{j}} \\
& =\frac{m \alpha_{v}}{\rho C_{p} \kappa_{s}} \sum_{\alpha=1}^{2}\left[\frac{h_{\alpha}}{m_{\alpha}}-\frac{C_{p}}{m \alpha_{v}}\left(\rho \frac{v_{\alpha}}{m_{\alpha}}\right)\right] \frac{\partial j_{\alpha j}}{\partial x_{j}} \\
& +\frac{m \alpha_{v}}{\rho C_{p} \kappa_{s}}\left[-\frac{\partial q_{\mathrm{IK} j}}{\partial x_{j}}+\tau_{i j} \frac{\partial u_{i}}{\partial x_{j}}\right]
\end{aligned}
$$

[Note that because only three thermodynamic variables are needed to specify a thermodynamic state, one of the equations in the set of Eqs. (15) and (17-19) is redundant.]

\section{Similarity Solution}

As discussed in the Introduction, mixing-layer DNS typically uses erf or tanh profiles as the basic flow. Although the basic flow solution should satisfy the conservation equations with the given boundary conditions, in many cases such a solution is not available because of the complicated form of the equations. In these situations, an approximate solution is considered appropriate. ${ }^{6}$ One such approximate solution is the similarity solution, consistent with experimental observations that, at large downstream distances, the spatial mixing layer becomes self-similar. ${ }^{23}$ The interest here is to elucidate if and how the RGNI similarity profiles differ from the incompressible or PG profiles, in view of the importance of the thermodynamic variables and/or the Soret and Dufour effects.

\section{A. Similarity Equations}

Similarity equations are here derived from Eqs. (16-19) by assuming one-dimensional flow (all variables being functions of $x_{2}$ and $t$ only), null spanwise velocity, $u_{3}=0$, and constant pressure, then neglecting the convective terms and considering that $\left(\partial u_{1} / \partial x_{2}\right)^{2} \gg\left(\frac{4}{3}\right)\left(\partial u_{2} / \partial x_{2}\right)^{2}$. This last assumption is made to allow the similarity transformation, with the expectation that, in the cross-stream direction, the variation of $u_{2}$ is considerably smaller than that of $u_{1}$. We choose the similarity variable as $\eta=t^{-1 / 2} x_{2}$ to satisfy

$$
\frac{\partial \eta}{\partial t}=f(\eta)\left(\frac{\partial \eta}{\partial x_{2}}\right)^{2}
$$

so that $u_{1}, T$, and $Y_{2}$ will be functions of $\eta$ only. This leads to the similarity equations for $u_{1}, T$, and $Y_{2}$ :

$$
\begin{aligned}
& \frac{1}{\rho} \frac{\mathrm{d}}{\mathrm{d} \eta}\left(\mu \frac{\mathrm{d} u_{1}}{\mathrm{~d} \eta}\right)+\frac{\eta}{2} \frac{\mathrm{d} u_{1}}{\mathrm{~d} \eta}=0 \\
& \frac{1}{\rho} \frac{\mathrm{d}}{\mathrm{d} \eta}\left(\rho D \alpha_{D} \frac{\mathrm{d} Y_{2}}{\mathrm{~d} \eta}+\alpha_{\mathrm{BK}} Y_{2} Y_{1} \frac{\rho D}{T} \frac{\mathrm{d} T}{\mathrm{~d} \eta}\right)+\frac{\eta}{2} \frac{\mathrm{d} Y_{2}}{\mathrm{~d} \eta}=0 \\
& \frac{m}{C_{p}}\left[\frac{1}{\rho} \frac{\mathrm{d}}{\mathrm{d} \eta}\left(\lambda_{\mathrm{IK}}^{\prime} \frac{\mathrm{d} T}{\mathrm{~d} \eta}+\alpha_{\mathrm{IK}} R_{u} T \frac{m}{m_{2} m_{1}} \rho D \alpha_{D} \frac{\mathrm{d} Y_{2}}{\mathrm{~d} \eta}\right)\right] \\
& \quad+\frac{m}{C_{p}}\left[\left(\frac{h_{, 2}}{m_{2}}-\frac{h_{, 1}}{m_{1}}\right) \frac{\eta}{2} \frac{\mathrm{d} Y_{2}}{\mathrm{~d} \eta}+\frac{\mu}{\rho}\left(\frac{\mathrm{d} u_{1}}{\mathrm{~d} \eta}\right)^{2}\right]+\frac{\eta}{2} \frac{\mathrm{d} T}{\mathrm{~d} \eta}=0
\end{aligned}
$$

and a nonsimilar equation for $u_{2}$ (from the pressure equation)

$$
\frac{\partial u_{2}}{\partial \eta}=-\frac{\eta}{2} \frac{1}{t^{1 / 2}}\left\{\alpha_{v} \frac{\mathrm{d} T}{\mathrm{~d} \eta}+\rho\left(\frac{v_{, 2}}{m_{2}}-\frac{v_{, 1}}{m_{1}}\right) \frac{\mathrm{d} Y_{2}}{\mathrm{~d} \eta}\right\}
$$

\section{B. Results}

The similarity equations are solved by using a fourth-order finite difference relaxation method. The range of $\eta$ depended on the species system and temperature conditions. The solution was verified to be independent of the resolution using 1601 and 3201 grid points. For ease of comparison, all profiles were scaled to have the same vorticity thickness $\delta_{\omega}$,

$$
\delta_{\omega}=\frac{\Delta U_{0}}{\left(\partial u_{1} / \partial x_{2}\right)_{\max }}=t^{1 / 2} \frac{\Delta U_{0}}{\left(\mathrm{~d} u_{1} / \mathrm{d} \eta\right)_{\max }}
$$

in the physical coordinate $\left(x_{2}=\eta t^{1 / 2}\right)$, from which the time $t$ was computed. At the selected $t,\left(\frac{4}{3}\right)\left(\partial u_{2} / \partial x_{2}\right)^{2}$ is two orders of magnitude smaller than $\left(\partial u_{1} / \partial x_{2}\right)^{2}$ for all simulations considered; therefore, the former term was justifiably neglected in the similarity analysis. Furthermore, the incompressiblesolution for $u_{1}$ is proportional to $\operatorname{erf}[\sqrt{ }(\rho / \mu) \eta / 2]=\operatorname{erf}\left[\sqrt{ }(\pi) x_{2} / \delta_{\omega}\right]$ :

$$
u_{1}\left(x_{2}\right)=u_{1}(-\infty)+U\left[\operatorname{erf}\left(\sqrt{\pi} x_{2} / \delta_{\omega}\right)+1\right]
$$

where the reference velocity is $U=\left[u_{1}(\infty)-u_{1}(-\infty)\right] / 2=$ $\Delta U_{0} / 2$

In Figs. 2 and 3 the solutions $u_{1}, Y_{2}$, and $T$ as functions of $\eta$ and $x_{2}$ are shown, obtained from simulations with $R e_{0}=800$ and $\delta_{\omega}=6.859 \times 10^{-3} \mathrm{~m}$. The complete freestream conditions are listed in Table 3 for heptane/nitrogen (HN) and for oxygen/ hydrogen $(\mathrm{OH})$. Computations at $R e_{0}=400$ revealed that the similarity profiles in the $x_{2}$ coordinate are independent of Reynolds number when all profiles are rescaled to have the same vorticity thickness. (The values $R e_{0}=400$ and $R e_{0}=800$ bracket the range used in the DNS of Refs. 14 and 15). All simulations are performed in a thermodynamic regime supercritical for the freestream fluid (see critical conditionsin Table 1). Because of the disparate speciessystem thermodynamics, it was not possible to match the density stratification of the $\mathrm{HN}$ and $\mathrm{OH}$ layers within the range of validity of their EOS and transport properties that also corresponds to regimes of practical interest. The solutions are compared either with $\operatorname{erf}[\sqrt{ }(\rho / \mu) \eta / 2]$, or with $\operatorname{erf}\left[\sqrt{ }(\pi) x_{2} / \delta_{\omega}\right]$.

For HN (Fig. 2), we note that all profiles have an inflection point and assume erf-like profiles in both coordinates, but none of the solutions conforms exactly to erf. When comparing the solutions in both coordinates, we note that the erf is fuller than all of the similarity solutions, with the $u_{1}$ profile being closest to erf. The $T$ profile is always more relaxed than that of $Y_{2}$, consistent with an effective Lewis number much larger than unity at supercritical conditions (see Ref. 24). The nonmonotonic behavior of $T$ near the heptane freestream region is due both to EOS effects and to $S c$ and $\operatorname{Pr}$ variations. For $\mathrm{OH}$ (Fig. 3), the $u_{1}$ and $Y_{2}$ profiles are similar to those of $\mathrm{HN}$ in being close to, but more relaxed than, the erf. However, the $T$ profile is fuller than the erf and is also fuller than the $u_{1}$ and $Y_{2}$ profiles. Also, nonmonotonic behavior is observed for the $T$ profile near both the oxygen and hydrogen freestreams. Becasue the hydrogen stream is close to a PG (Table 3 and Fig. 4), the $T$ behavior is a manifestation of the varying $S c$ and $P r$.

To ascertain the degree of mixture nonideality and real-gas effects, $Z$ and $\alpha_{D}$ for the conditions of Figs. 2 and 3 are plotted in Fig. 4, where $Z=p /\left(\rho T R_{u} / m\right)$ is the compression factor portraying departures from the PG behavior, that is, $Z=1$. The OHa layer is close to a perfect gas, ideal mixture (which would have $\alpha_{D}=1$ ), whereas $\mathrm{OHb}$ and $\mathrm{OHc}$ are close to ideal mixtures but not so close to being PG. The HNb and HNc layers show significant departures from mixture ideality and PG behavior, with $Z$ as low as 0.5 and $\alpha_{D}$ as low as 0.6 .

This analysis shows that the real-gas EOS play an essential role in depicting the physics of the problem. Also, realistic $S c$ and $P r$ are important in determining the details of the similarity profiles. It should be kept in mind that real-gas effects involve not only the 


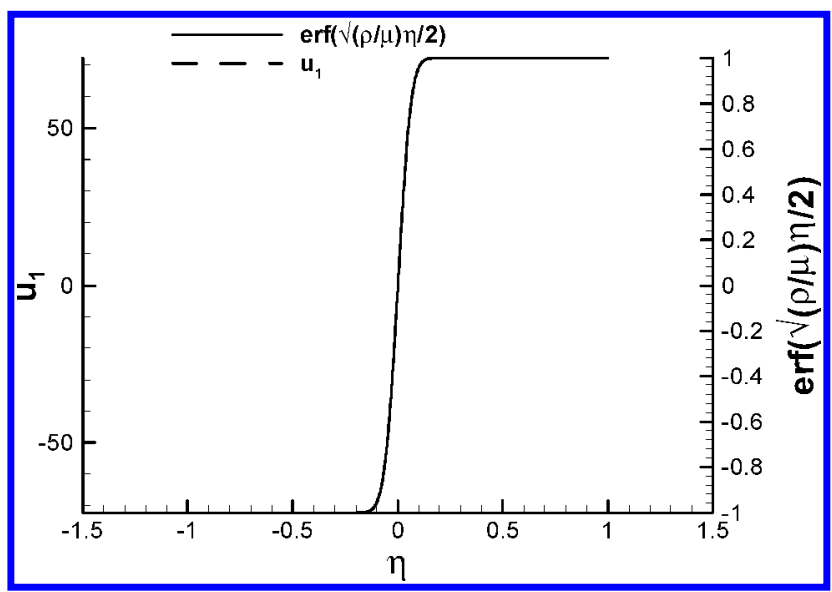

a)

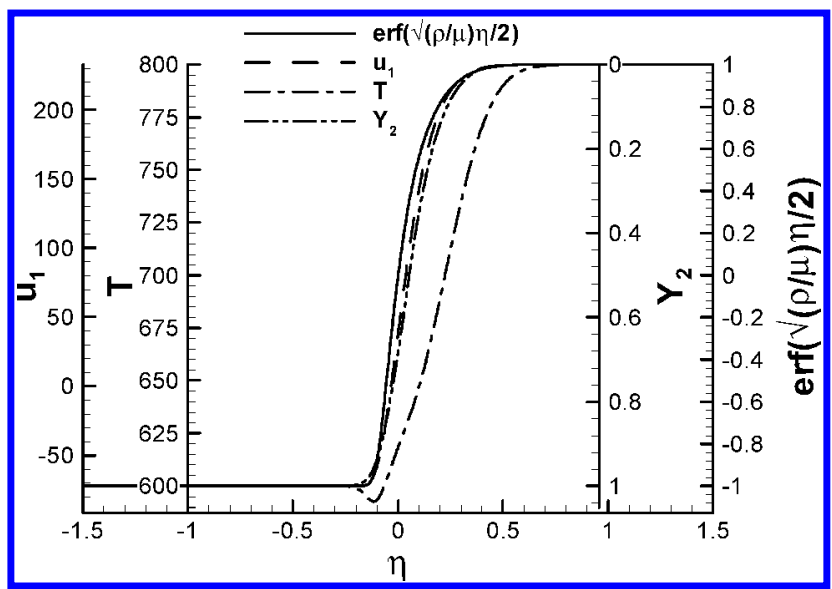

c)

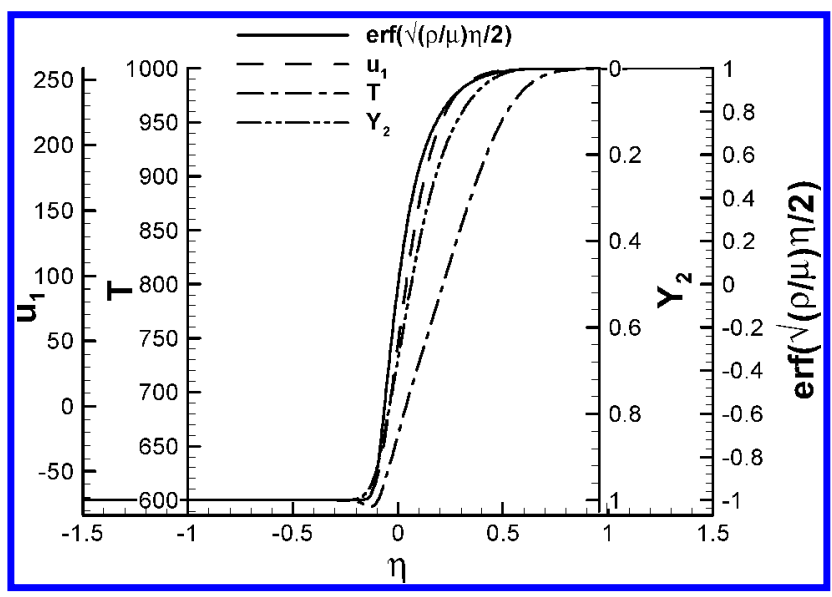

e)

Fig. 2 Similarity profiles of $u_{1}$ (meters per second), $T$ (degrees Kelvin), and $Y_{2}$ for (a and b) $\mathrm{HNa}$, (c and d) $\mathrm{HNb}$, and (e and f) HNc vs $\left(\mathrm{a}, \mathrm{c}\right.$, and e) the similarity variable and $(\mathrm{b}, \mathrm{d}$, and $\mathrm{f})$ the physical variables; $R e_{0}=800$.
EOS, $S c$ and $P r$, but also mixture nonideality and Soret and Dufour effects (thermal diffusion). All of these effects need to be retained for thermodynamic consistency in real-gas behavior. Because all of these effects strongly interact, PG/ideal mixture simulations with constant diffusion coefficients, that is, constant $S c$ and $P r$, are inappropriate for revealing the separate impact of the EOS, that is, without accounting for transport properties.

\section{Three-Dimensional Inviscid Temporal Stability Analysis}

In conducting the temporal stability analysis of the supercritical mixing layer, our interest is focused on determining the most unsta-

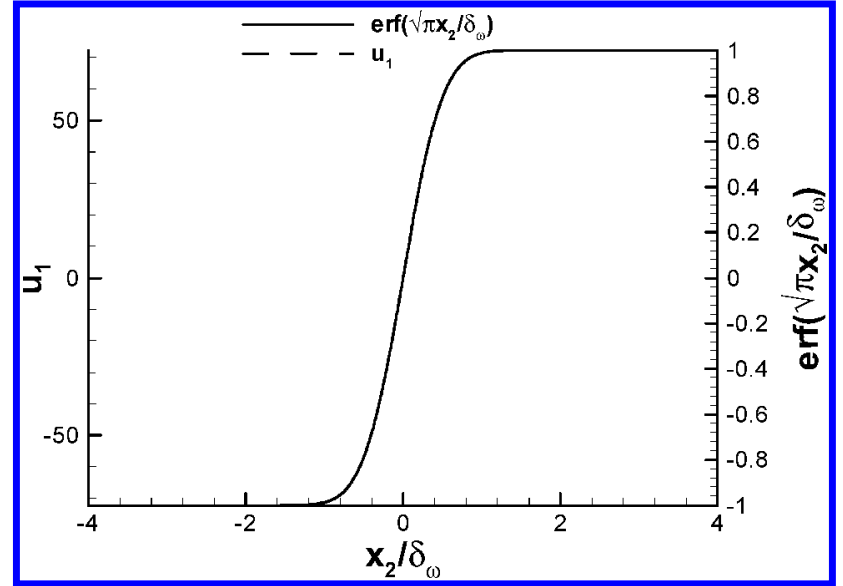

b)

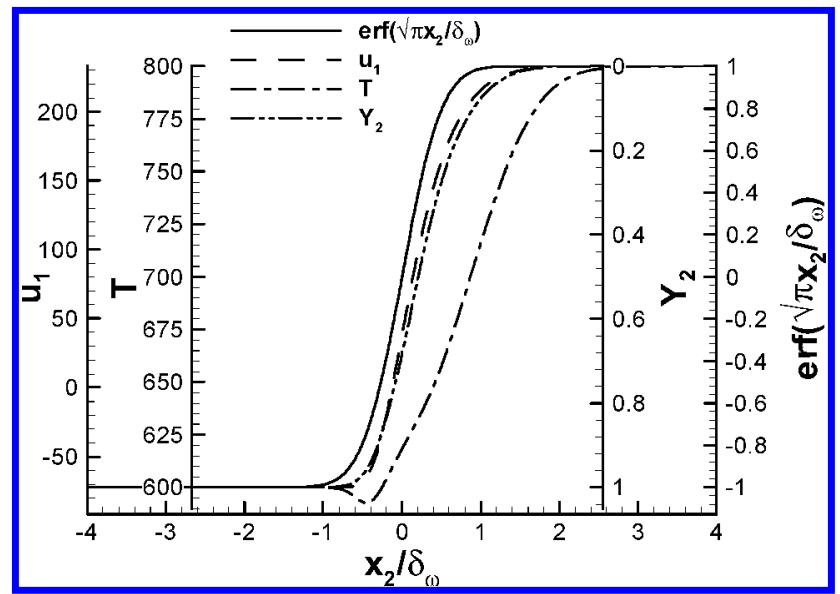

d)

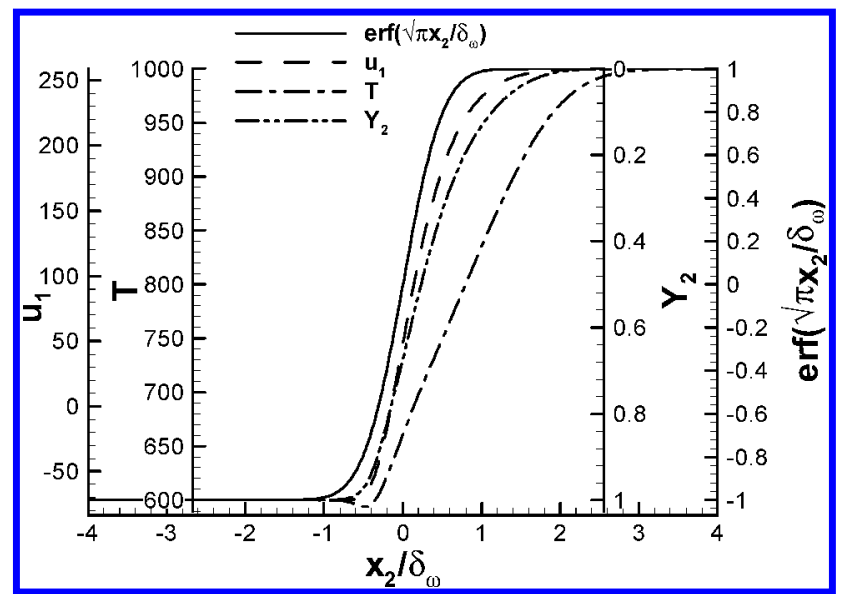

ble wavelength for future simulations of turbulent three-dimensional layers. When turbulence is achieved, the molecular diffusional processes are unimportant from the viewpoint of the large-scale dynamics, and the memory of the initial conditions is entirely lost. It is well known that the stability characteristics of flows with a single inflection point are much less sensitive to the form of the basic flow than are other flows (Ref. 6, p. 211), and therefore, mixing layers use erf or tanh mean profiles. This representationis also justified by the results of Shin and Ferziger, ${ }^{9}$ who studied both the temporal and spatial stability of compressible flows with and without heat release and found that for nonreacting conditions the basic flow has a single inflection point and that the effect of the initial velocity profile on 


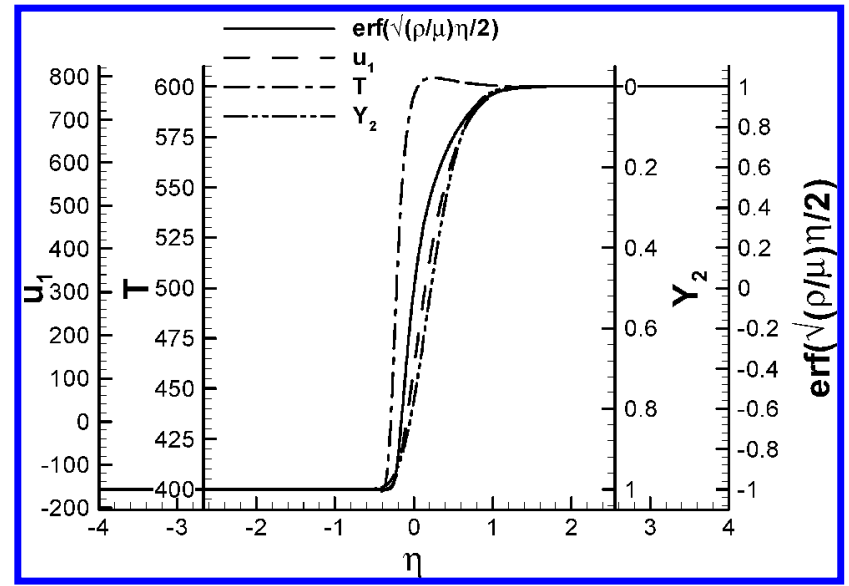

a)

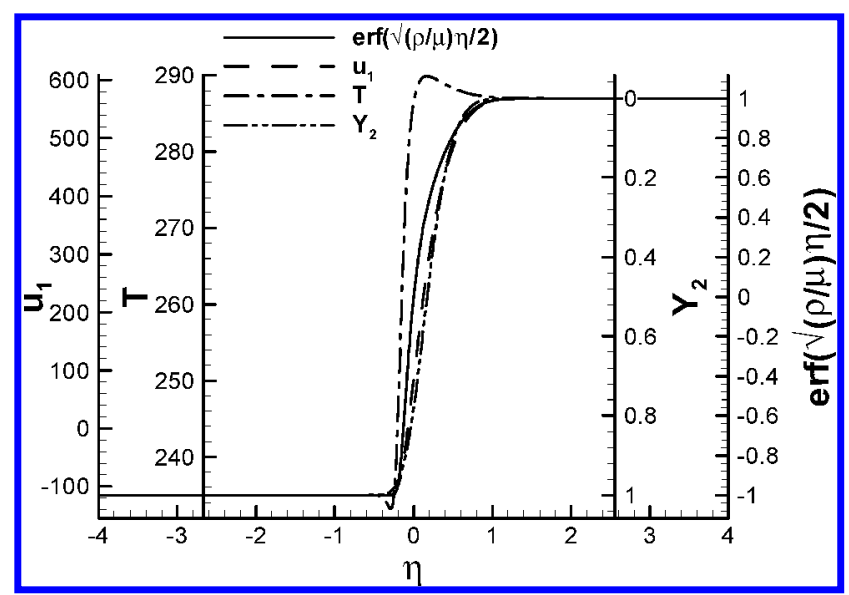

c)

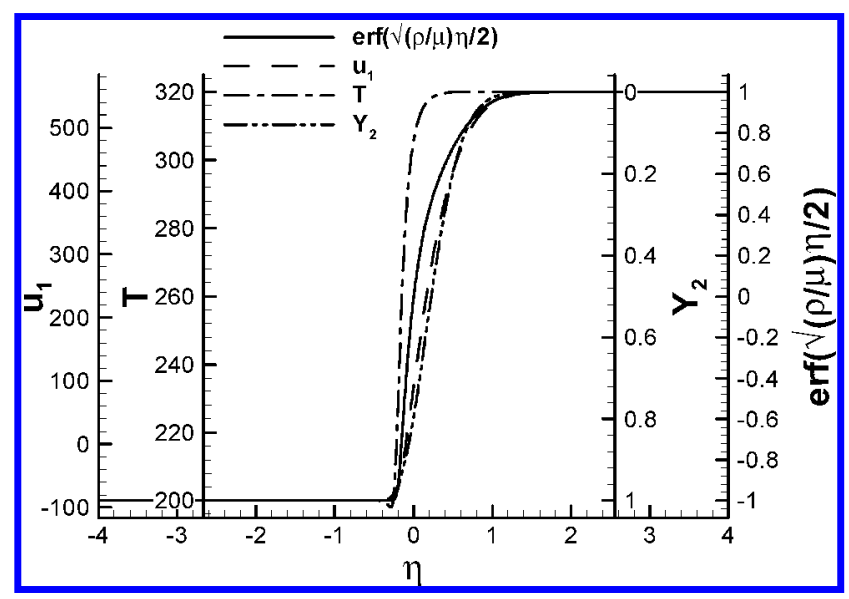

e)

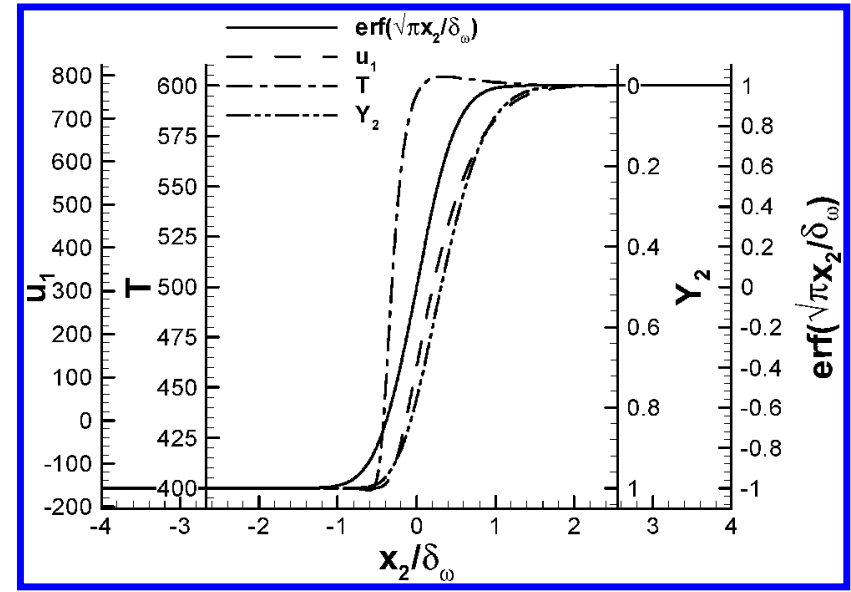

b)

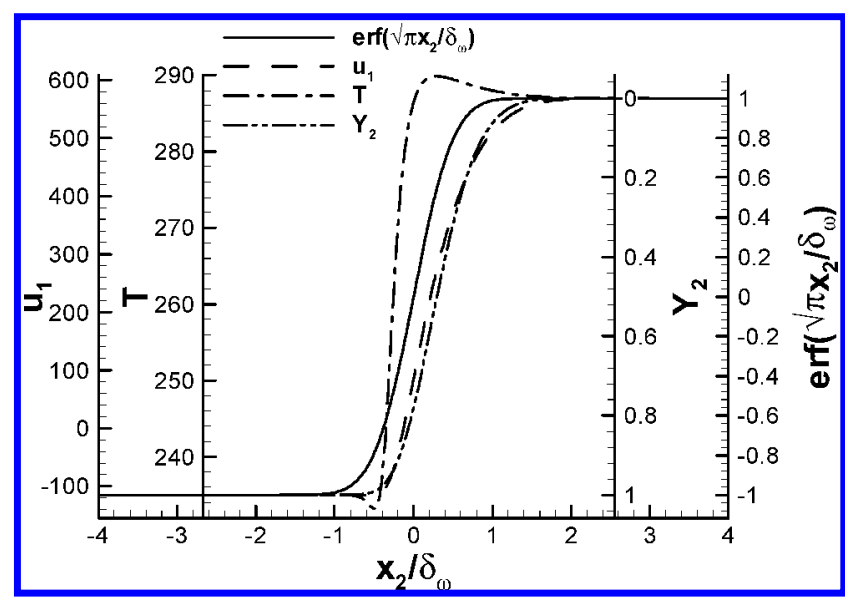

d)

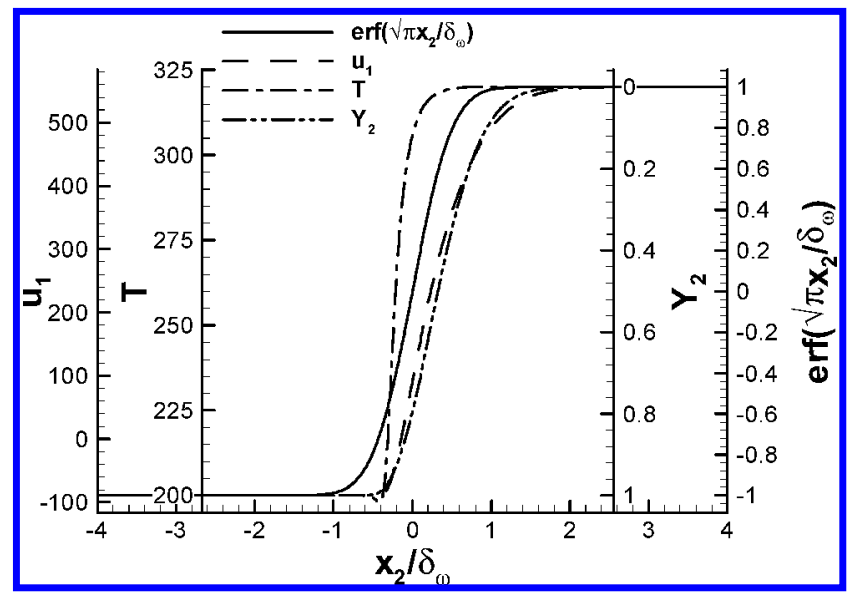

Fig. 3 Similarity profiles of $u_{1}$ (meters per second), $T$ (degrees Kelvin), and $Y_{2}$ for (a and b) OHa, (c and d) OHb, and (e and f) OHc vs $\left(\mathrm{a}, \mathrm{c}\right.$, and e) the similarity variable and $(\mathrm{b}, \mathrm{d}$, and $\mathrm{f})$ the physical variables; $\boldsymbol{R e}_{0}=\mathbf{8 0 0}$.

the growth rate is very small. (The rates obtained with the similarity solution were compared to those obtained with a tanh profile.) Similar results were found (for the erf) by Lu and Lele ${ }^{10}$ from a spatial stability analysis in the range of freestream temperature ratios studied here.

Following well-accepted methods for studying flow instabilities (e.g., Refs. 6, 9, and 11), we conduct a linear, inviscid analysis of the conservation equations. [Viscous effects are of higher order because $R e \geq O\left(10^{2}\right)$ and are, therefore, confined to the basic flow.] The present stability analysis uses, for the mean flow, either the similarity profiles (Figs. 2 and 3) or erf profiles. The interest is in determining whether the stability characteristics of RGNI layers are similar to well-studied incompressible or PG layers.

\section{A. Equations}

The point of departure for the temporal stability analysis is the primitive form of the equations (Sec. II.E). The neglect of the viscous terms uncouples Eq. (17) from the remaining equations (and, therefore, the $Y_{2}$ equation is not considered), and leaves the threedimensional inviscid equations for $\rho, u_{i}$, and $p$. The perturbed flow variables $\psi=\left(\rho, u_{i}, p\right)$ are given by $\psi=\bar{\psi}+\Delta \psi$, where the overbar denotes the mean flow variables, which are functions of $x_{2}$ only, with the perturbations given by

$$
\Delta \psi=\hat{\psi}\left(x_{2}\right) \exp \left[i \alpha\left(x_{1} \cos \phi+x_{3} \sin \phi-c t\right)\right]
$$

where the caret denotes the perturbation amplitudes and, for temporal analysis, $\alpha$ is real and $c$ is complex. The functional form 


\begin{tabular}{lcccccc}
\hline \hline Case & $\mathrm{HNa}$ & $\mathrm{HNb}$ & $\mathrm{HNc}$ & $\mathrm{OHa}$ & $\mathrm{OHb}$ & $\mathrm{OHc}$ \\
\hline$\rho_{2} / \rho_{1}$ & 1 & 10.38 & 12.93 & 24.40 & 24.51 & 38.87 \\
$\Delta U_{0}=U_{1}-U_{2}$ & 144.406 & 305.09 & 330.73 & 932.73 & 683.90 & 645.53 \\
$\left|\rho_{2} U_{2}\right| /\left|\rho_{1} U_{1}\right|$ & 1 & 3.222 & 3.596 & 4.940 & 4.951 & 6.235 \\
Stream 1 species & $\mathrm{C}_{7} \mathrm{H}_{16}$ & $\mathrm{~N}_{2}$ & $\mathrm{~N}_{2}$ & $\mathrm{H}_{2}$ & $\mathrm{H}_{2}$ & $\mathrm{H}_{2}$ \\
Stream 2 species & $\mathrm{C}_{7} \mathrm{H}_{16}$ & $\mathrm{C}_{7} \mathrm{H}_{16}$ & $\mathrm{C}_{7} \mathrm{H}_{16}$ & $\mathrm{O}_{2}$ & $\mathrm{O}_{2}$ & $\mathrm{O}_{2}$ \\
$U_{1}, \mathrm{~m} / \mathrm{s}$ & 72.203 & 2332.83 & 258.77 & 775.71 & 568.97 & 556.30 \\
$U_{2}, \mathrm{~m} / \mathrm{s}$ & -72.203 & -72.268 & -71.954 & -157.02 & -114.93 & -89.225 \\
$a_{s 1}, \mathrm{~m} / \mathrm{s}$ & 180.508 & 582.59 & 644.714 & 1915.38 & 1381.95 & 1447.53 \\
$a_{s 2}, \mathrm{~m} / \mathrm{s}$ & 180.508 & 180.508 & 180.508 & 397.52 & 295.99 & 214.64 \\
$\rho_{1}, \mathrm{~kg} / \mathrm{m}^{3}$ & 259.46 & 24.997 & 20.060 & 3.965 & 8.050 & 7.2526 \\
$\rho_{2}, \mathrm{~kg} / \mathrm{m}^{3}$ & 259.46 & 259.46 & 259.46 & 96.764 & 197.32 & 281.93 \\
$T_{1}, \mathrm{~K}$ & 600 & 800 & 1000 & 600 & 287 & 320 \\
$T_{2}, \mathrm{~K}$ & 600 & 600 & 600 & 400 & 235 & 200 \\
$\mathrm{Stream} 1 Y_{2}$ & 1 & 0 & 0 & 0 & 0 & 0 \\
$\mathrm{Stream} 2 Y_{2}$ & 1 & 1 & 1 & 1 & 1 & 1 \\
$Z_{1}$ & 0.4707 & 1.0243 & 1.0211 & 1.0327 & 1.0634 & 1.0586 \\
$Z_{2}$ & 0.4707 & 0.4707 & 0.4707 & 1.0075 & 0.9382 & 0.6916 \\
$p_{0}$, atm & 60 & 60 & 60 & 100 & 100 & 100 \\
$p_{r 1}=p / p_{c 1}$ & 2.22 & 1.79 & 1.79 & 7.89 & 7.89 & 7.89 \\
$p_{r 2}=p / p_{c 2}$ & 2.22 & 2.22 & 2.22 & 2.01 & 2.01 & 2.01 \\
\hline \hline
\end{tabular}

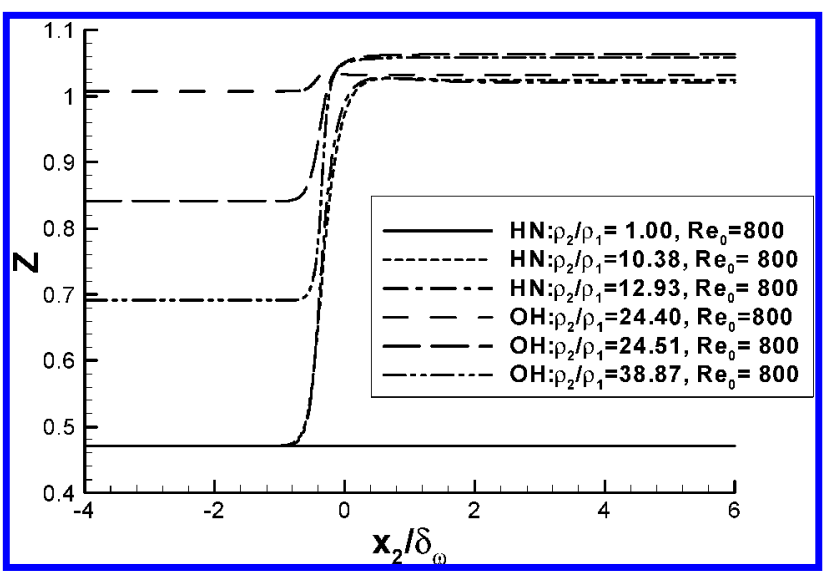

a)

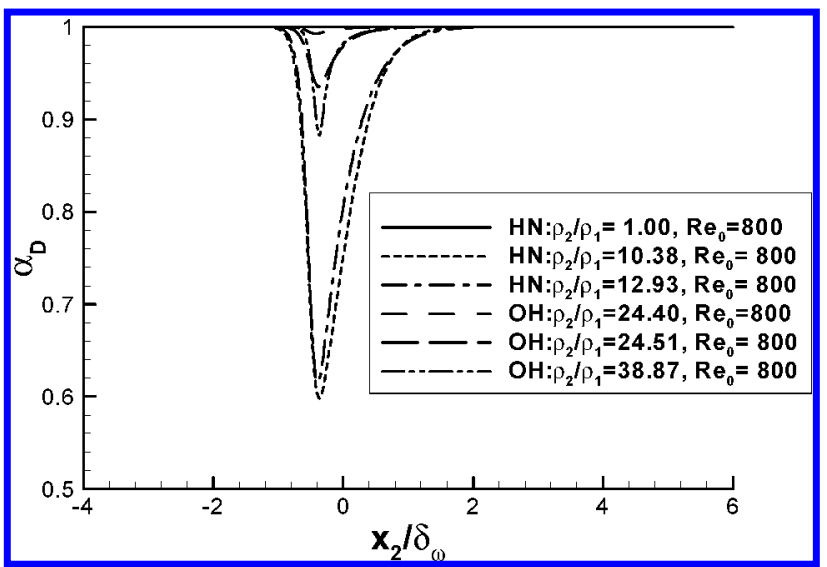

b)

Fig. 4 Similarity profiles for all layers of a) $Z$ and b) $\alpha_{D}$.

of the perturbation in the $\left(x_{1}, x_{3}\right)$ plane is chosen so that the unit vector makes an angle $\phi$ with the streamwise direction. The streamwise and spanwise wavelengths are $\lambda_{1}=2 \pi /(\alpha \cos \phi)$ and $\lambda_{3}=2 \pi /(\alpha \sin \phi)$, respectively $\left(\lambda_{3} / \lambda_{1}=1 / \tan \phi\right)$. Within the protocol of the stability analysis, the physicalquantities are obtained by taking the real part of the complex quantities. Substituting the expressionsfor the flow variables, linearizing, neglecting perturbations in the fluid properties (including in $a_{s}$ ), and assuming streamwise mean flow, that is, $\bar{u}_{2}=\bar{u}_{3}=0$, with uniform mean pressure $\bar{p}=p_{0}$ yields coupled differential equations for the perturbations, which when further manipulated become

$$
\frac{\mathrm{d}^{2} \hat{p}}{\mathrm{~d} x_{2}^{2}}-\left(\frac{1}{\bar{\rho}} \frac{\mathrm{d} \bar{\rho}}{\mathrm{d} x_{2}}+\frac{2 \cos \phi}{\left(\bar{u}_{1} \cos \phi-c\right)} \frac{\mathrm{d} \bar{u}_{1}}{\mathrm{~d} x_{2}}\right) \frac{\mathrm{d} \hat{p}}{\mathrm{~d} x_{2}}-\left(1-M^{2}\right) \alpha^{2} \hat{p}=0
$$

$$
M\left(x_{2}\right)=\frac{\bar{u}_{1} \cos \phi-c}{a_{s}}
$$

with boundary conditions

$$
x_{2} \rightarrow \pm \infty: \hat{p}=0
$$

The other perturbations are given by

$$
\begin{gathered}
\hat{u}_{2}=-\frac{1}{\left(\bar{u}_{1} \cos \phi-c\right) i \alpha} \frac{1}{\bar{\rho}} \frac{\mathrm{d} \hat{p}}{\mathrm{~d} x_{2}} \\
\hat{u}_{1}=-\frac{1}{\left(\bar{u}_{1} \cos \phi-c\right) i \alpha}\left[\hat{u}_{2} \frac{\mathrm{d} \bar{u}_{1}}{\mathrm{~d} x_{2}}+\frac{\hat{p}}{\bar{\rho}} i \alpha \cos \phi\right] \\
\hat{u}_{3}=-\frac{1}{\left(\bar{u}_{1} \cos \phi-c\right)} \frac{\hat{p}}{\bar{\rho}} \sin \phi \\
\hat{\rho}=\frac{\hat{p}}{a_{s}^{2}}-\frac{1}{\left(\bar{u}_{1} \cos \phi-c\right) i \alpha} \frac{\mathrm{d} \bar{\rho}}{\mathrm{d} x_{2}} \hat{u}_{2}
\end{gathered}
$$

Equation (28) shows that the three-dimensional instability problem with mean flow $\bar{u}_{1}$ corresponds to a two-dimensional $(\phi=0)$ problem with mean flow $\bar{u}_{1} \cos \phi$, so in general the threedimensional eigenvalue for mean flow $\bar{u}_{1}$ cannot be obtained by solving a two-dimensional problem using the same mean flow $\bar{u}_{1}$.

The incompressibleform of the equations can be found by setting $a_{s} \rightarrow \infty$ and $\bar{\rho}$ constant, leading to $M=0$ and $\mathrm{d} \bar{\rho} / \mathrm{d} x_{2}=0$, in which case Eq. (28) becomes

$$
\frac{\mathrm{d}^{2} \hat{p}}{\mathrm{~d} x_{2}^{2}}-\frac{2}{\left(\bar{u}_{1}-c / \cos \phi\right)} \frac{\mathrm{d} \bar{u}_{1}}{\mathrm{~d} x_{2}} \frac{\mathrm{d} \hat{p}}{\mathrm{~d} x_{2}}-\alpha^{2} \hat{p}=0
$$

and, thus, for incompressible flow, given the two-dimensional $(\phi=0)$ eigenvalue $c$ for mean flow $\bar{u}_{1}$, the three-dimensionaleigenvalue for mean flow $\bar{u}_{1}$ is simply $c / \cos \phi$. In other words, the threedimensional compressible stability problem depends on $u_{1} \cos \phi$, 
and therefore, as in the incompressible case, any oblique wave is related to a two-dimensional wave with a different mean flow magnitude. In the incompressible case, the eigenvalue of the threedimensional problem can be determined from the eigenvalue of the two-dimensionalproblem, which is not the case for the compressible problem, due to the $\left(u_{1} \cos \phi-c\right) / a_{s}$ term.

\section{B. Numerical Method}

Equation (28) specifies an eigenvalue problem: Given $\bar{u}_{1}, \bar{\rho}, \alpha, a_{s}$, and $\phi$, a solution must be found for $\hat{p}$ and $c$. The eigenvalue problem is solved numerically by matching the asymptotic solutions at finite

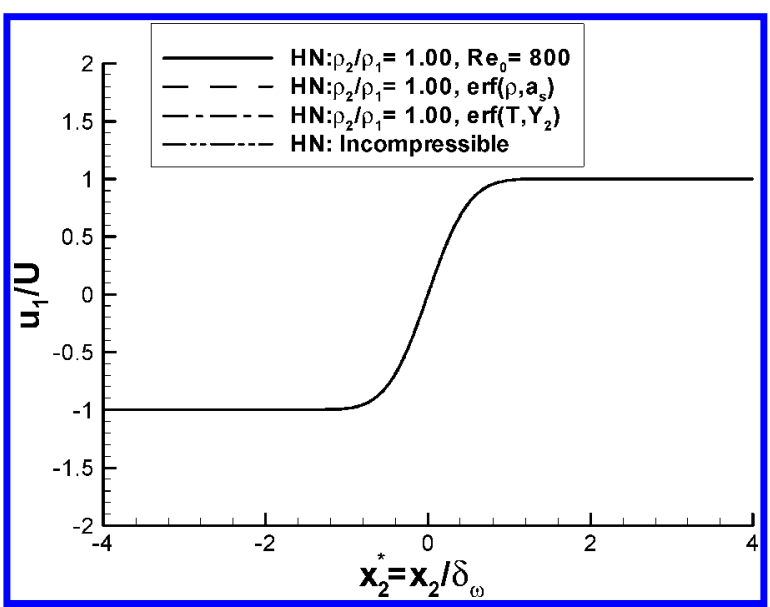

a)

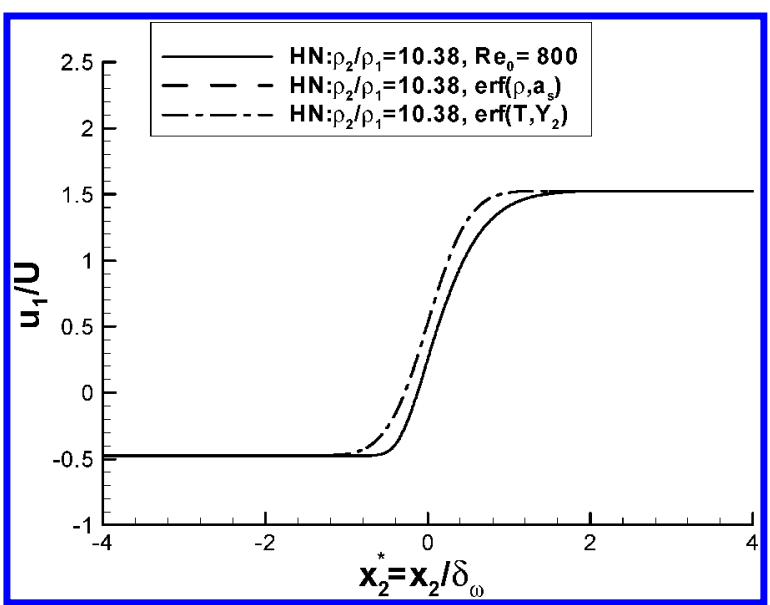

c)

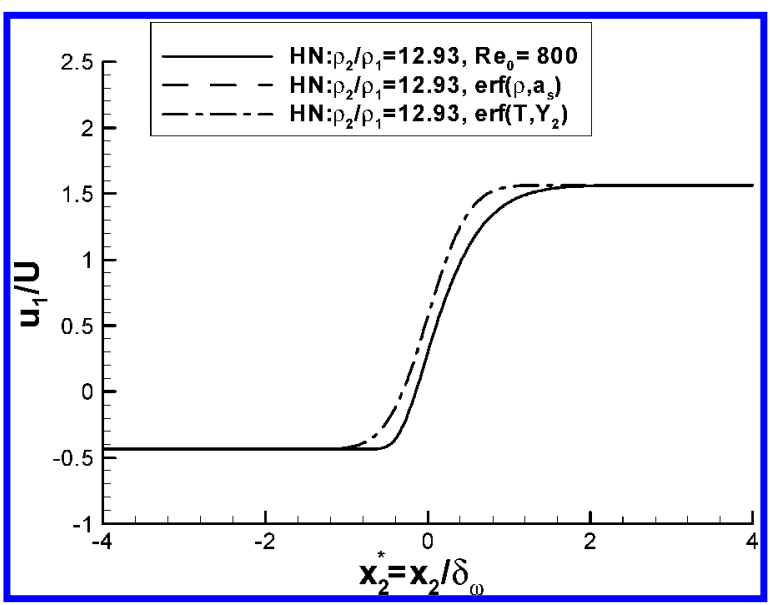

e) $x_{2}$, which are derived by setting the boundaries in a region where the mean flow gradients are null so that $M$ is constant. Denoting $\varepsilon=\sqrt{ }\left(1-M^{2}\right)$ (a complex constant with value $\varepsilon^{-}$at $-\infty$ and value $\varepsilon^{+}$at $\left.+\infty\right)$, then

$$
x_{2} \rightarrow \pm \infty: \frac{\mathrm{d}^{2} \hat{p}}{\mathrm{~d} x_{2}^{2}}-\left(\varepsilon^{ \pm} \alpha\right)^{2} \hat{p}=0
$$

$$
\begin{gathered}
x_{2} \rightarrow-\infty: \hat{p}=a_{1} \exp \left(\varepsilon^{-} \alpha x_{2}\right)+a_{2} \exp \left(-\varepsilon^{-} \alpha x_{2}\right) \\
x_{2} \rightarrow \infty: \hat{p}=b_{1} \exp \left(-\varepsilon^{+} \alpha x_{2}\right)+b_{2} \exp \left(\varepsilon^{+} \alpha x_{2}\right)
\end{gathered}
$$

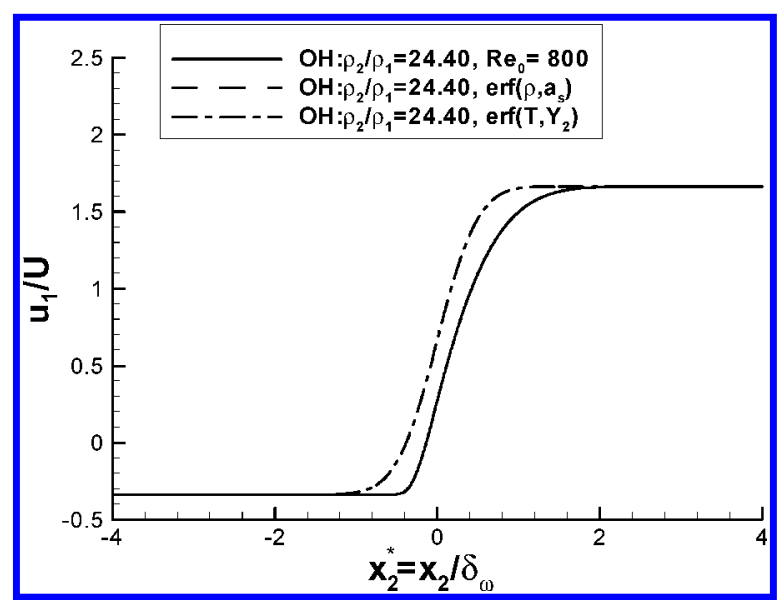

b)

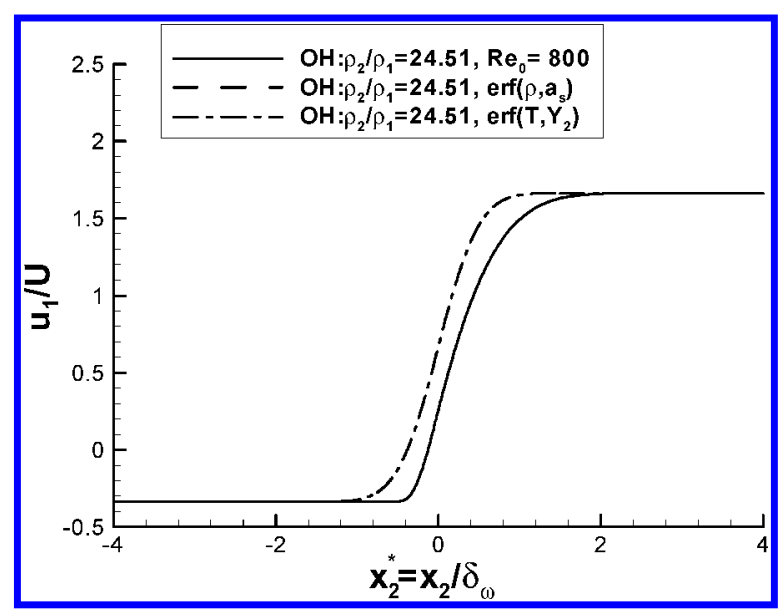

d)

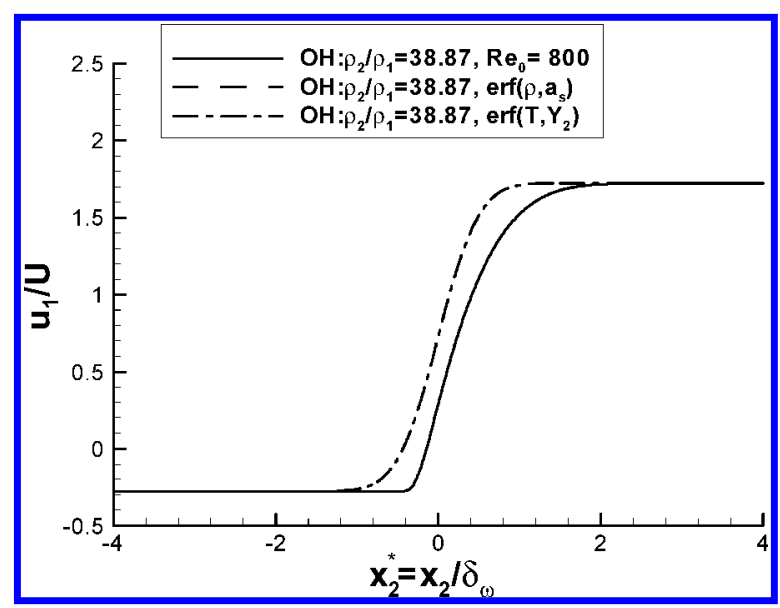

f)

Fig. 5 Mean streamwise velocity profiles for all layers, where the $u_{1}$ profiles for erf $\left(\rho, a_{s}\right)$ and erf $\left(T, Y_{2}\right)$ are identical for each layer: a) HNa, b) OHa, c) $\mathrm{HNb}$, d) $\mathrm{OHb}$, e) $\mathrm{HNc}$, and f) OHc. 
where $a_{1}, a_{2}, b_{1}$, and $b_{2}$ are complex constants, which depending on $\varepsilon^{ \pm}$, may be set to zero to keep $\hat{p}$ bounded to satisfy the boundary conditions (30). Because any multiple of a solution is also a solution, the nonzero values of $a_{1}$ and $a_{2}$ are set equal to $(1,-1)$. The values of $b_{1}$ and $b_{2}$ are eliminated from the boundary condition specification by relating $\hat{p}$ and its derivative.

\section{Two-Dimensional Results of the Stability Analysis}

The ordinary differential equation for $\hat{p}$ [Eq. (28) with $\phi=0$ ] is solved in dimensionless form, with the reference length and velocity for nondimensionalization being $\delta_{\omega}$ and
$U=\Delta U_{0} / 2$. The results obtained with three mean profile types are compared:

1) For $R e_{0}=800$, similarity profile of Sec. III is used.

2) For erf( $\left.\rho, a_{s}\right)$, the mean velocity is given by an erf [Eq. (26)], where $\bar{u}_{1}(\infty)=U_{1}$ and $\bar{u}_{1}(-\infty)=U_{2}$ as given by Eq. (13). The same erf form is used for $a_{s}$ and $1 / \bar{\rho}$. This form for $\bar{\rho}$ and $a_{s}$ does not depend on the EOS, and because $\bar{\rho}$ and $a_{s}$ appear explicitly in the stability problem [Eq. (28)], specifying their form allows us to conveniently take the constant density and incompressible limits.

3) For erf $\left(T, Y_{2}\right)$, the mean velocity is given by an erf [Eq. (26)]. The same erf form is used for $\bar{T}$ and $\bar{Y}_{2}$; along with a uniform

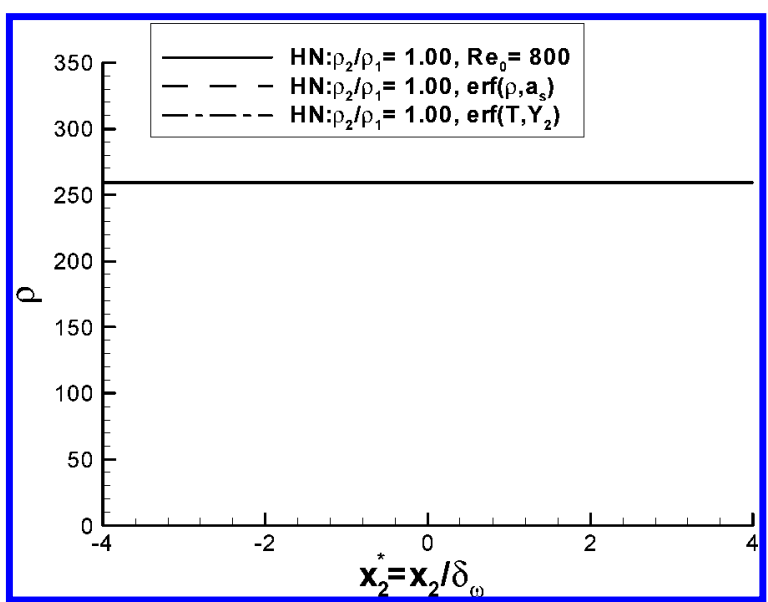

a)

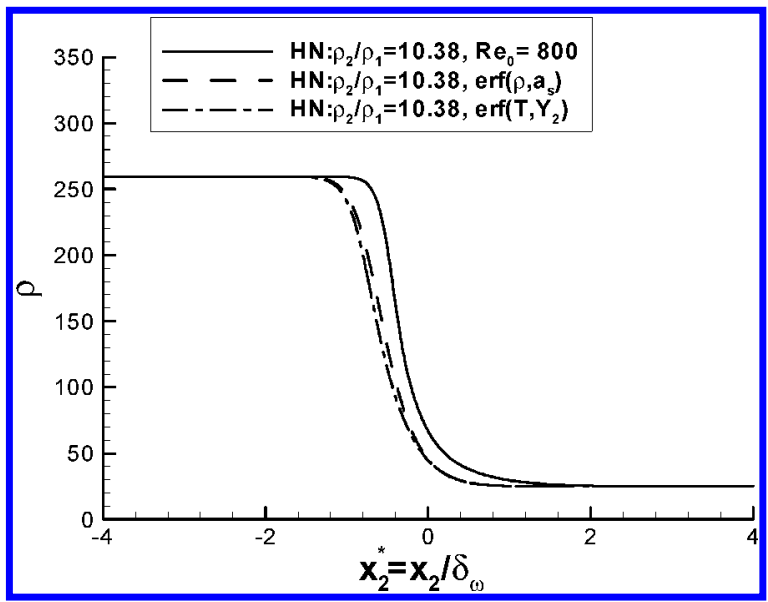

c)

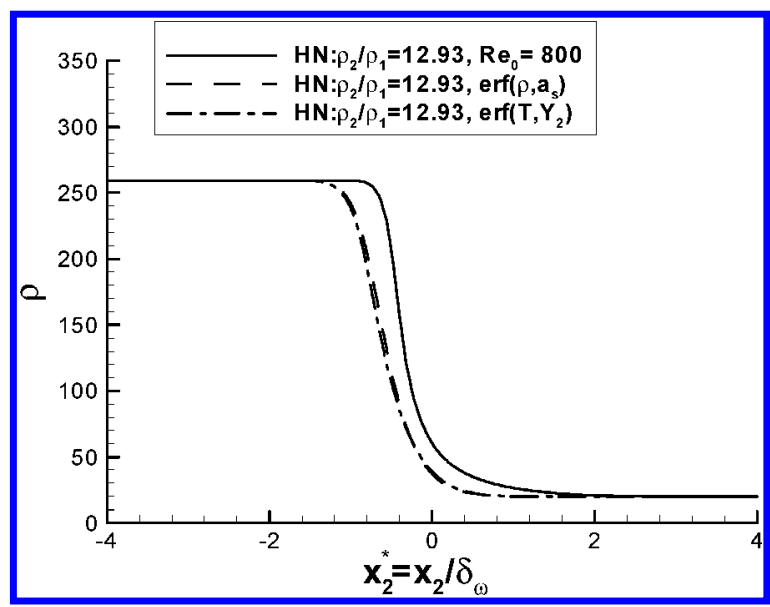

e)

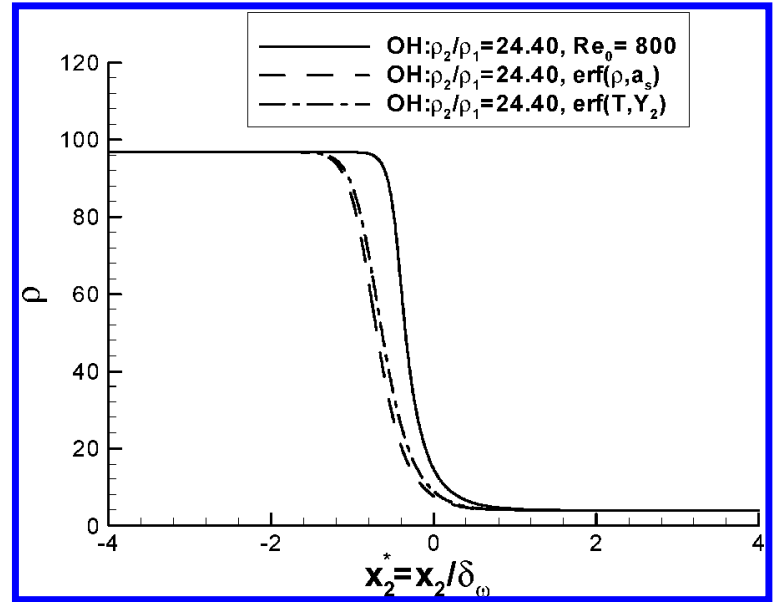

b)

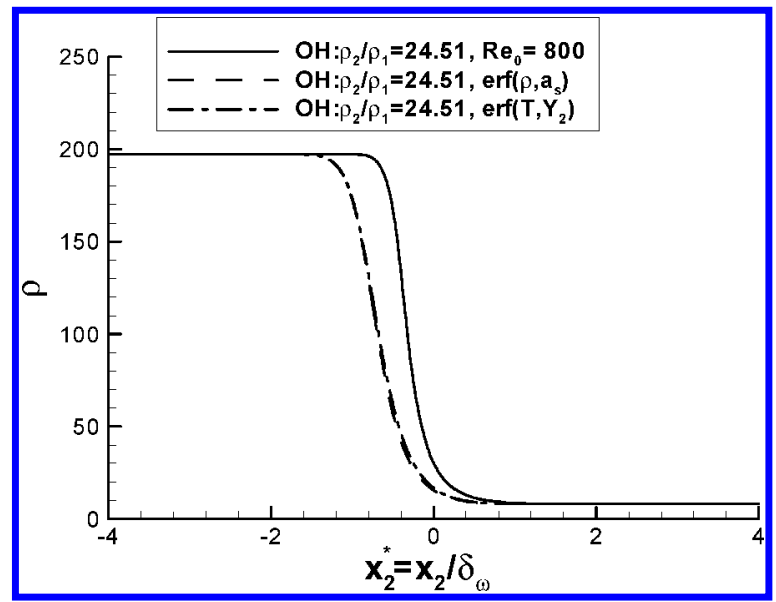

d)

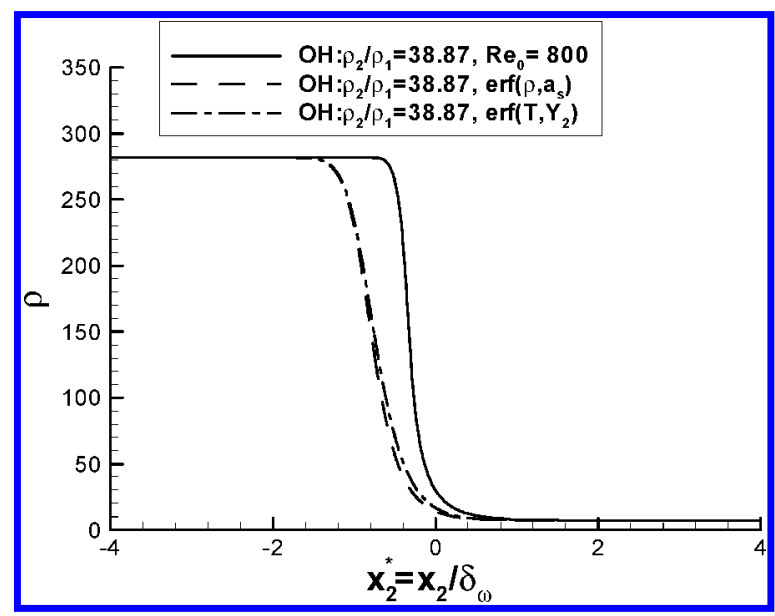

f)

Fig. 6 Mean density (kilograms per cubic meter) profiles for all layers in Fig. 5. 
pressure, the equation of state is used to calculate $a_{s}$ and $\bar{\rho}$ from $\bar{T}$ and $\bar{Y}_{2}$.

The freestream values of the mean quantities used in the calculations are given in Table 3, whereas Figs. 5-7 show the mean profiles. As mentioned in Sec. III, the similarity $\bar{u}_{1}$ (Fig. 5) is more relaxed than erf. Also, for both $\mathrm{HN}$ and $\mathrm{OH}$ layers, the two types of erf profiles lead to $\rho$ profiles (Fig. 6) that are nearly alike. The erf-based $a_{s}$ profiles are very close for $\mathrm{HN}$, whereas for $\mathrm{OH}$ layers, some differences are evident (Fig. 7).

Equation (28) is solved using a fourth-orderRunge-Kutta integrator over the range $x_{2, \text { min }}^{*} \leq x_{2}^{*} \equiv x_{2} / \delta_{\omega} \leq x_{2, \text { max }}^{*}$, starting at $x_{2, \text { min }}^{*}$ and marching to $x_{2, \max }^{*}$, where $x_{2, \min }^{*}$ and $x_{2, \max }^{*}$ are selected large enough

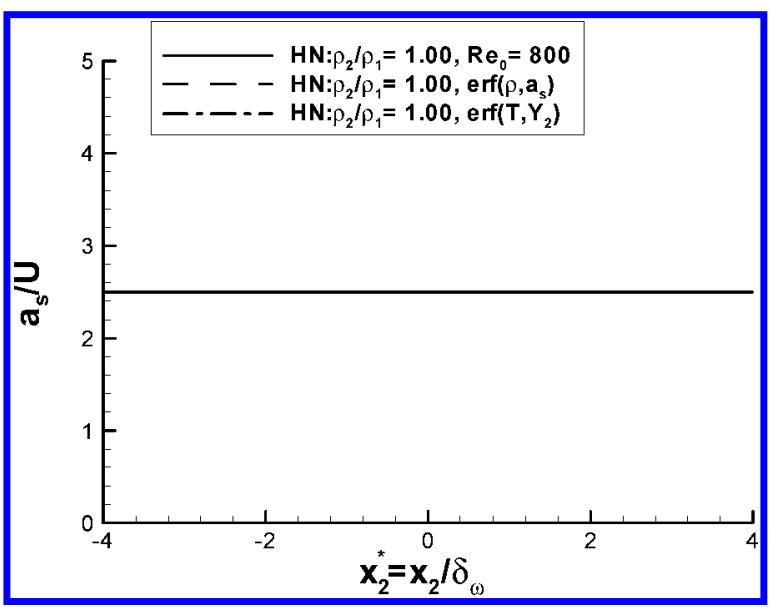

a)

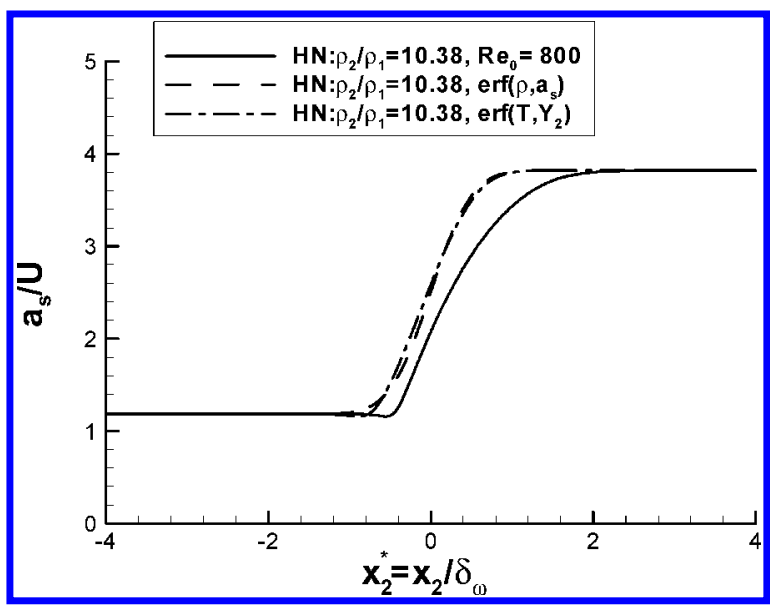

c)

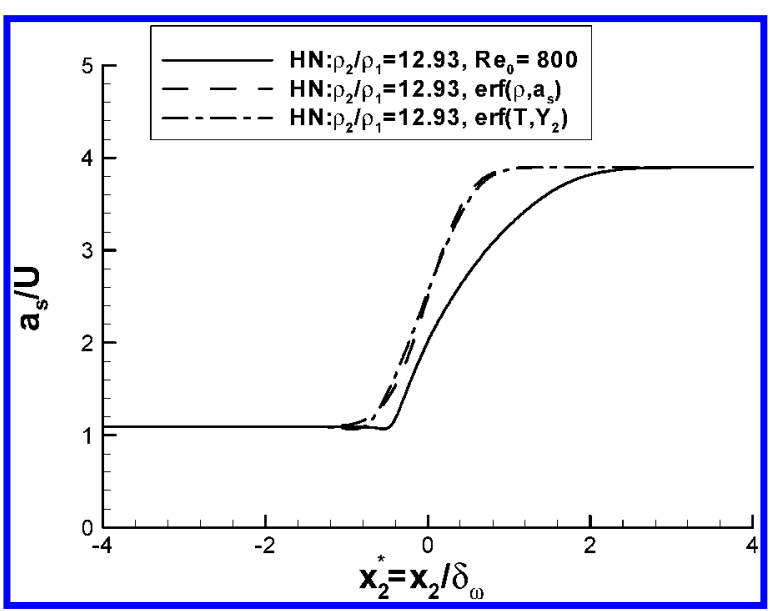

e) to be in a region where the asymptotic solution [Eqs. (36-38)] is valid. The range of $x_{2}^{*}$ is less than for the similarity solution because excessively large values of $x_{2, \min }^{*}$ and $x_{2, \max }^{*}$ create numerical errors that destabilize the integration of Eq. (28). The starting values for the perturbation and its derivative at $x_{2, \text { min }}^{*}$ are obtained from the asymptotic solution, Eqs. (36-38). A steepest-descent optimizer is used to solve the eigenvalue problem for each $\alpha$ by adjusting $c$ so that the asymptotic relations between the perturbation and its derivative at $x_{2, \max }^{*}$ [derived from Eq. (38)] are satisfied. The integration interval has twice the grid spacing of the similarity solution; the results obtained are the same when doubling the grid resolution, or when doubling the integrationinterval. For marching in $\alpha$, the initial

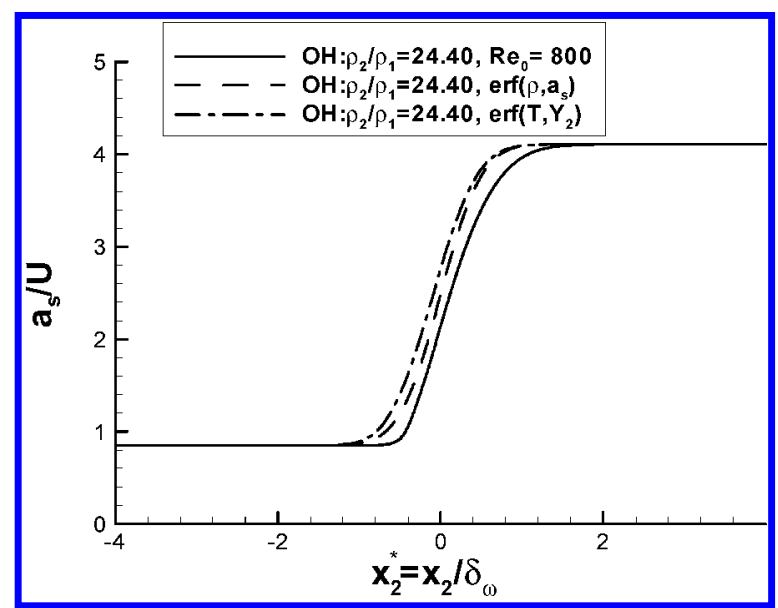

b)

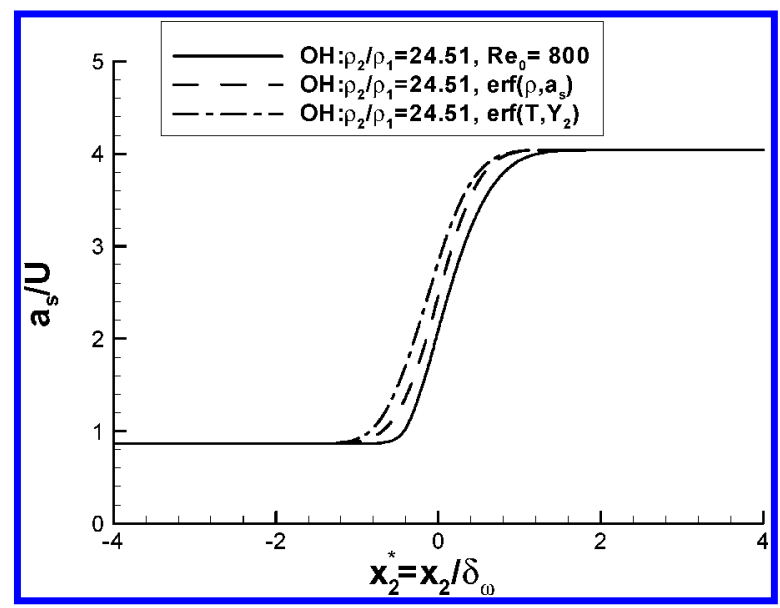

d)

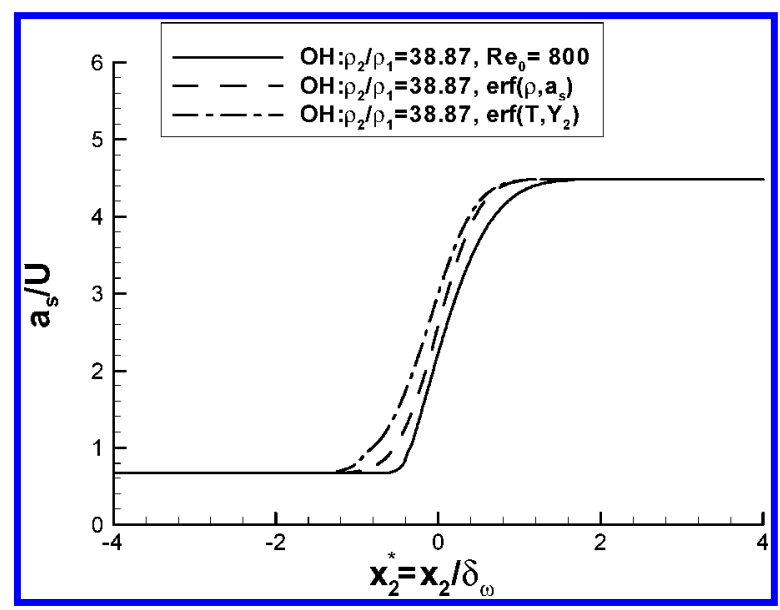

f)

Fig. 7 Mean speed of sound profiles for all layers in Fig. 5. 
Table 4 Most unstable and shortest unstable wavelengths for $\mathrm{C}_{7} \mathrm{H}_{16} / \mathrm{N}_{2}$ layers, two-dimensional analysis (see Table 3 for freestream conditions $\mathrm{HNa}, \mathrm{HNb}$, and $\mathrm{HNc}$ )

\begin{tabular}{|c|c|c|c|c|c|c|c|c|c|c|}
\hline \multirow[b]{2}{*}{ Case } & \multirow[b]{2}{*}{ Profile type } & \multirow[b]{2}{*}{$\rho_{2} / \rho_{1}$} & \multicolumn{4}{|c|}{ Most unstable wavelength } & \multicolumn{4}{|c|}{ Shortest unstable wavelength } \\
\hline & & & $\alpha \delta_{\omega}$ & $\alpha \delta_{\omega} c_{i} / U$ & $c_{r} / U$ & $\lambda_{1} / \delta_{\omega}$ & $\alpha \delta_{\omega}$ & $\alpha \delta_{\omega} c_{i} / U$ & $c_{r} / U$ & $\lambda_{1} / \delta_{\omega}$ \\
\hline $\mathrm{HNa}$ & Incompressible & 1 & 0.86 & 0.3830 & -0.0000 & 7.31 & 1.79 & 0.0357 & -0.0000 & 3.51 \\
\hline $\mathrm{HNa}$ & $\operatorname{erf}\left(\rho, a_{s}\right)$ & 1 & 0.80 & 0.3151 & -0.0000 & 7.85 & 1.62 & 0.0324 & -0.0000 & 3.88 \\
\hline $\mathrm{HNa}$ & $\operatorname{erf}\left(T, Y_{2}\right)$ & 1 & 0.80 & 0.3151 & -0.0000 & 7.85 & 1.62 & 0.0324 & -0.0000 & 3.88 \\
\hline $\mathrm{HNa}$ & Similarity, $R e_{0}=400$ & 1 & 0.80 & 0.3151 & -0.0000 & 7.85 & 1.61 & 0.0392 & -0.0000 & 3.91 \\
\hline $\mathrm{HNa}$ & Similarity, $R e_{0}=800$ & 1 & 0.80 & 0.3151 & -0.0000 & 7.85 & 1.61 & 0.0392 & -0.0000 & 3.91 \\
\hline $\mathrm{HNb}$ & $\operatorname{erf}\left(\rho, a_{s}\right)$ & 10.38 & 0.74 & 0.2607 & 0.5369 & 8.49 & 1.48 & 0.0340 & 0.5265 & 4.24 \\
\hline $\mathrm{HNb}$ & $\operatorname{erf}\left(T, Y_{2}\right)$ & 10.38 & 0.74 & 0.2606 & 0.5376 & 8.49 & 1.48 & 0.0360 & 0.5267 & 4.24 \\
\hline $\mathrm{HNb}$ & Similarity, $R e_{0}=800$ & 10.38 & 0.88 & 0.2342 & -0.0841 & 7.14 & 1.97 & 0.0809 & -0.1546 & 3.19 \\
\hline $\mathrm{HNc}$ & $\operatorname{erf}\left(\rho, a_{s}\right)$ & 12.93 & 0.72 & 0.2481 & 0.5854 & 8.72 & 1.41 & 0.0567 & 0.5650 & 4.46 \\
\hline $\mathrm{HNc}$ & $\operatorname{erf}\left(T, Y_{2}\right)$ & 12.93 & 0.72 & 0.2475 & 0.5866 & 8.73 & 1.38 & 0.0631 & 0.5668 & 4.55 \\
\hline $\mathrm{HNc}$ & Similarity, $R e_{0}=800$ & 12.93 & 0.87 & 0.2227 & -0.0681 & 7.22 & 1.88 & 0.0994 & 0.1358 & 3.34 \\
\hline
\end{tabular}

Table 5 Most unstable and shortest unstable wavelengths for $\mathrm{O}_{2} / \mathrm{H}_{2}$ layers, two-dimensional analysis (see Table 3 for freestream conditions $\mathrm{OHa}, \mathrm{OHb}$, and $\mathrm{OHc}$ )

\begin{tabular}{|c|c|c|c|c|c|c|c|c|c|c|}
\hline \multirow[b]{2}{*}{ Case } & \multirow[b]{2}{*}{ Profile type } & \multirow[b]{2}{*}{$\rho_{2} / \rho_{1}$} & \multicolumn{4}{|c|}{ Most unstable wavelength } & \multicolumn{4}{|c|}{ Shortest unstable wavelength } \\
\hline & & & $\alpha \delta_{\omega}$ & $\alpha \delta_{\omega} c_{i} / U$ & $c_{r} / U$ & $\lambda_{1} / \delta_{\omega}$ & $\alpha \delta_{\omega}$ & $\alpha \delta_{\omega} c_{i} / U$ & $c_{r} / U$ & $\lambda_{1} / \delta_{\omega}$ \\
\hline $\mathrm{OHa}$ & $\operatorname{erf}\left(\rho, a_{s}\right)$ & 24.40 & 0.60 & 0.1283 & -0.0713 & 10.47 & 1.33 & 0.0279 & -0.1032 & 4.72 \\
\hline $\mathrm{OHa}$ & $\operatorname{erf}\left(T, Y_{2}\right)$ & 24.40 & 0.74 & 0.1545 & -0.0712 & 8.49 & 1.58 & 0.0325 & -0.0853 & 3.98 \\
\hline $\mathrm{OHa}$ & Similarity, $R e_{0}=400$ & 24.40 & 1.33 & 0.2218 & -0.1151 & 4.72 & 2.53 & 0.1420 & -0.1370 & 2.48 \\
\hline $\mathrm{OHa}$ & Similarity, $R e_{0}=800$ & 24.40 & 1.33 & 0.2218 & -0.1151 & 4.72 & 2.53 & 0.1420 & -0.1370 & 2.48 \\
\hline $\mathrm{OHb}$ & $\operatorname{erf}\left(\rho, a_{s}\right)$ & 24.51 & 0.61 & 0.1280 & -0.0704 & 10.30 & 1.46 & 0.0044 & -0.1092 & 4.30 \\
\hline $\mathrm{OHb}$ & $\operatorname{erf}\left(T, Y_{2}\right)$ & 24.51 & 0.68 & 0.1420 & -0.0692 & 9.24 & 1.43 & 0.0298 & -0.0856 & 4.39 \\
\hline $\mathrm{OHb}$ & Similarity, $R e_{0}=800$ & 24.51 & 1.43 & 0.2283 & -0.1223 & 4.39 & 2.27 & 0.1908 & -0.1330 & 2.77 \\
\hline $\mathrm{OHc}$ & $\operatorname{erf}\left(\rho, a_{s}\right)$ & 38.87 & 0.55 & 0.0985 & -0.0672 & 11.42 & 1.25 & 0.0235 & -0.0983 & 5.03 \\
\hline $\mathrm{OHc}$ & $\operatorname{erf}\left(T, Y_{2}\right)$ & 38.87 & 0.71 & 0.1247 & -0.0613 & 8.85 & 1.45 & -0.0630 & 0.032 & 4.33 \\
\hline $\mathrm{OHc}$ & Similarity, $R e_{0}=800$ & 38.87 & 1.67 & 0.2008 & -0.1251 & 3.76 & 2.33 & 0.1878 & -0.1327 & 2.70 \\
\hline
\end{tabular}

guess for $c$ is the $\alpha=0$ analytical solution derived in Appendix B. The derivation of conditions on $c$ similar to the Howard's semicircle theorem for incompressible flows (e.g., Ref. 6) is also presented in Appendix B.

Figures 8 and 9 show the nondimensional growth rate, $\alpha c_{i} \delta_{\omega} / U$, vs the wave number for the cases in Table 3. Additionally, in Fig. 8a is the incompressible solution [obtained by the compressible formulation using $\bar{\rho}(\infty)=\bar{\rho}(-\infty)$ and $a_{s}=10^{6}$ and confirmed by a separate solution of Eq. (35)]. Tables 4 and 5 show the values of the most unstable wavelength (the wavelength with the maximum growth rate $\alpha c_{i}$ ), as well as the shortest (obtained) unstable wavelength for each case. From Fig. 8, which shows the effect of mean profile for each layer, it is clear that the growth rates of the layers are independent of profile shape at small wave numbers (long wavelengths). At shorter wavelengths, the growth rate is sensitive to the profile shape, with significantly higher growth rates for the similarity profile. The stability curves of the two $\mathrm{HN}$ erf profile types overlap, whereas those for $\mathrm{OH}$ do not, showing the EOS effect through $a_{s}$. The EOS effect is also seen in comparing the constant density and incompressibleflow results; the incompressibleflow has higher growth rate and wider range of unstable wave numbers. From Table 4, the most unstable wavelength is longer for the constant density than the incompressible flows. The incompressible flow most unstable wavelength, $\lambda_{1} / \delta_{\omega}=7.31$, compares favorably with the value of 7.29 used by Moser and Rogers. ${ }^{4}$ Figure 8 , in conjunction with Figs. 5-7, shows that there is not a straightforward relation between $\bar{u}_{1}, \bar{\rho}$, and $\bar{a}_{s}$ profile shapes and the resulting stability curve. This is because the three profiles interact to determine the stability characteristics, and therefore, detailed calculations rather than visual inspection are required to quantify the stability features of the flow.

In Fig. 9, the stability curves of Fig. 8 are replotted to illustrate the density stratification and species system effect. Note that the growth rate of each stratified flow is smaller than that of the constant density flow and that, as the stratification decreases, the stability curve tends toward the constant density one, as it should. Comparing stability curves for the same type of profile in each of Figs. 9a, 9b, and 9c, we note that they do not have maxima at the same wave number and that the wave number correspondingto the most unstable wavelength increases with decreasing stratification.

\section{Three-Dimensional Results of the Stability Analysis}

Similar to the two-dimensional study, we seek to investigate the stability curve and search for the most unstable wavelength. The solution of the stability equation (28) involves determining the eigenvalue $c$ for given values of $\alpha$ and $\phi(\alpha>0,0 \leq \phi<\pi / 2)$. The threedimensional eigenvalue problem is solved in a similar manner to the two-dimensional problem, but with the parameter $\phi$. Typically in three-dimensionalsimulations, it is desired to minimize both the streamwise and the spanwise extent of the domain and, thereby, reduce computational costs. (Because three-dimensional simulations are intrinsically different from two-dimensional simulations, it is not surprising that the corresponding stability analyses might be differently applied.) Therefore, we seek unstable solutions with $\phi$ as large as possible for a given value of $\alpha$. From the geometry, for a given $\alpha$, if the streamwise extent of the domain is decreased, the spanwise extent must be increased, or for a given $\phi$, if the streamwise extent of the domain is decreased, the spanwise extent must also be decreased. However, the optimal domain size cannot be determined entirely from geometric considerations because in this eigenvalue problem only certain combinations of $\alpha$ and $\phi$ will lead to flow instability. In fact for each $\alpha$, we have a range of unstable $\phi, 0 \leq \phi \leq \phi_{\max }$, and for each $\phi$, we have a range of unstable $\alpha$, $0<\alpha \leq \alpha_{\max }$.

The region of unstable three-dimensional solutions can be estimated from that of the unstable two-dimensional solutions by considering $\alpha_{\max }$ of the two-dimensional problem, $\alpha_{2 \mathrm{D}, \max }$, and noting that, for a given wave number of the three-dimensional problem, $\alpha$, its two-dimensional component is $\alpha_{1}=\alpha \cos \phi$, and therefore, $\phi=\cos ^{-1}\left(\alpha_{1} / \alpha\right)$. Because for instability neither $\alpha_{1}$ 


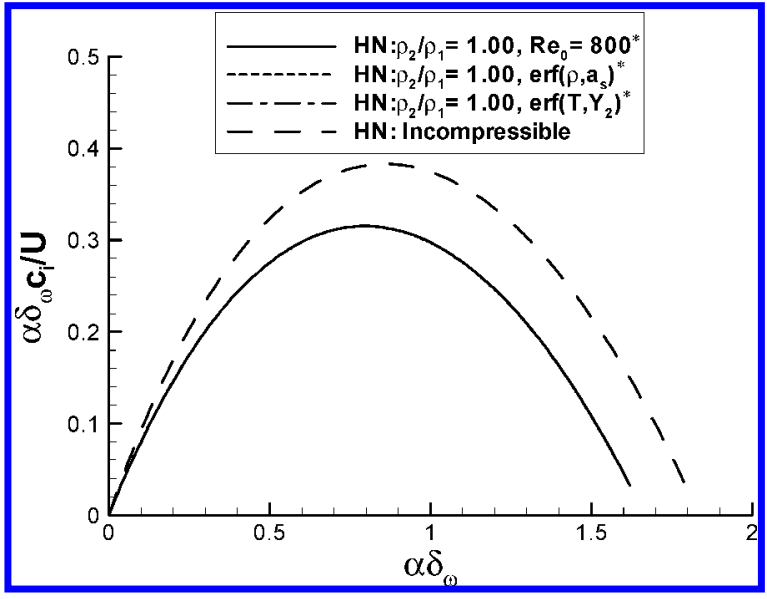

a)

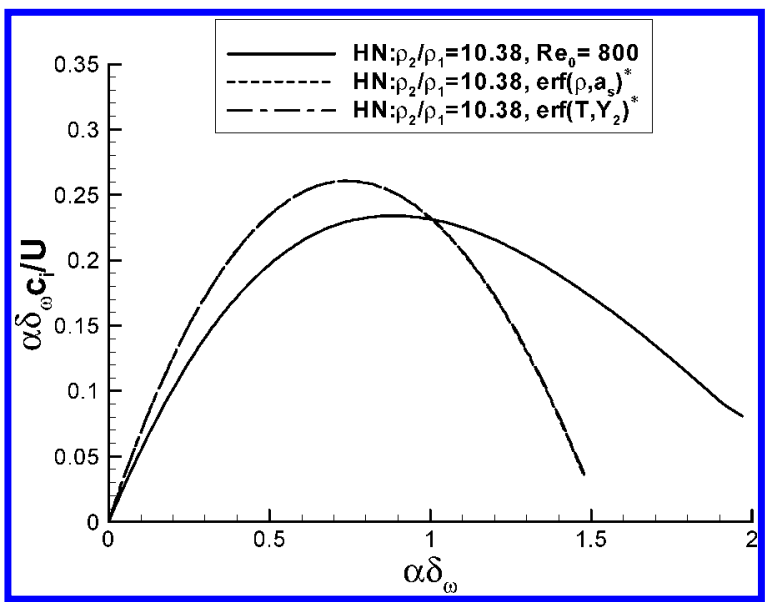

c)

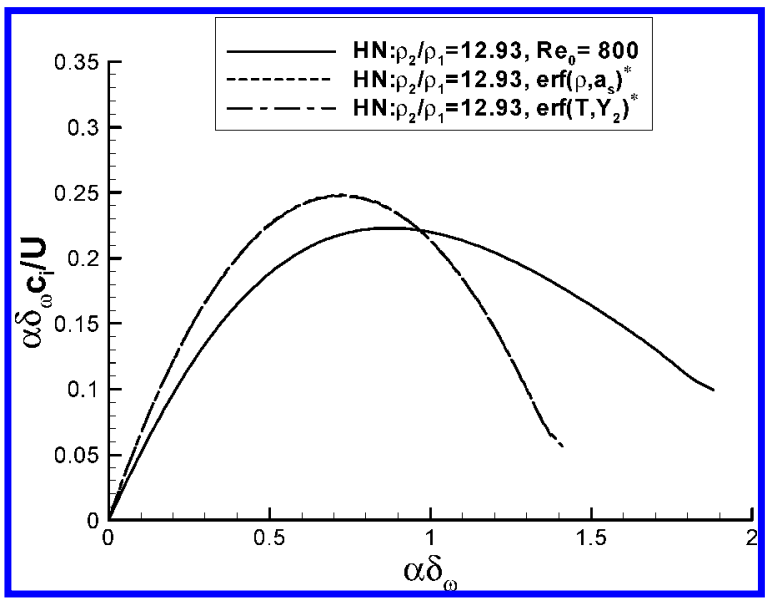

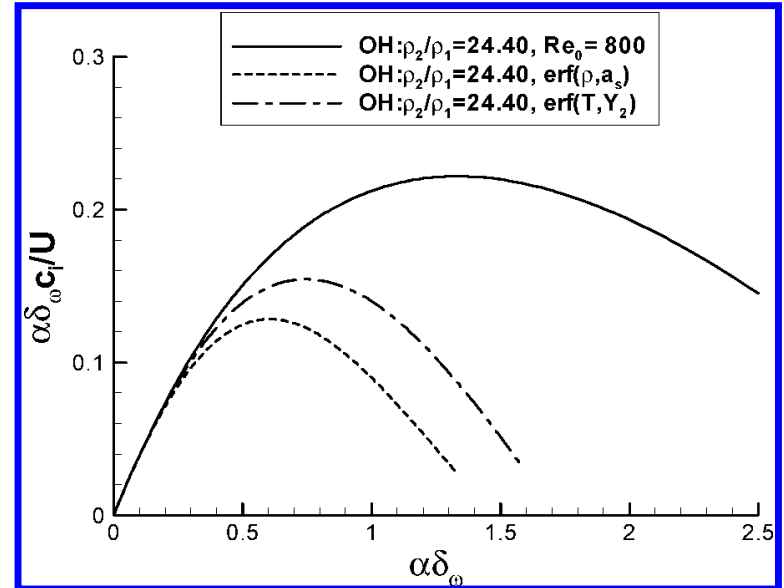

b)

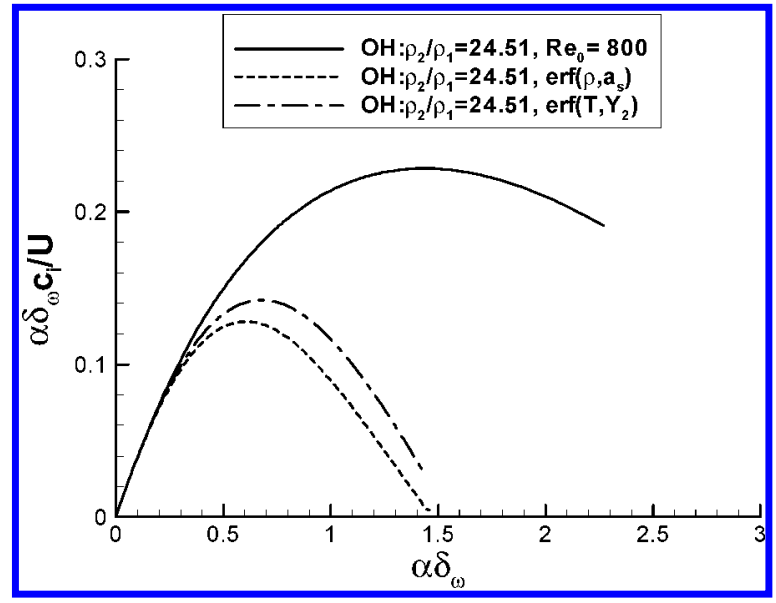

d)

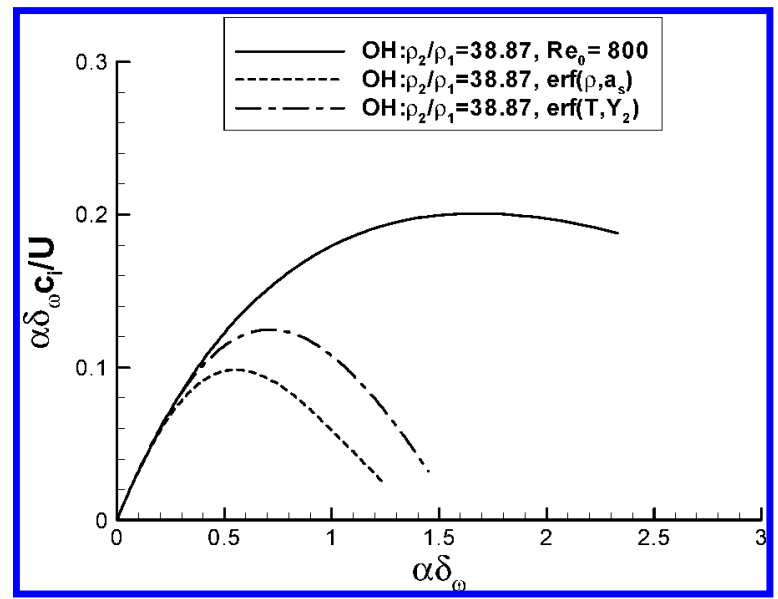

Fig. 8 Stability curves showing the effect of $u_{1}, \rho$, and $a_{s}$ mean profile shape for a) HNa, b) OHa, c) HNb, d) OHb, e) HNc, and f) OHc: $\star$, overlapping curves.

nor $\alpha$ may exceed $\alpha_{2 \mathrm{D}, \max }$, we have $\phi_{\max }\left(\alpha_{1}\right)=\cos ^{-1}\left(\alpha_{1} / \alpha_{2 \mathrm{D}, \max }\right)$ and $\alpha_{1, \max }(\phi)=\alpha_{2 \mathrm{D}, \max } \cos \phi$. In particular, $\alpha_{1}=\alpha_{2 \mathrm{D}, \max }$ implies $\phi_{\max }=0$, resulting in $\lambda_{3}$ being infinite; on the other hand, we could not find any purely spanwise $(\phi=\pi / 2)$ modes. In the context of applications to three-dimensional flow simulations, this implies that the ability to use a shorter wavelength in the streamwise direction for the purpose of achieving pairing and rollup will be offset by the need to use a longer wavelength in the spanwise direction. This finding may explain why, although the streamwise perturbationused in most shear layer simulations corresponds to an eigenvalue solution, the spanwise perturbation (cf., Moser and Rogers ${ }^{4}$ ) is heuris- tic and without equivalently established validity. In other words, many DNS, although using most unstable two-dimensional modes in two-dimensional calculations, do not use most unstable threedimensional modes for three-dimensional calculations, but rather the most unstable two-dimensional mode plus heuristic spanwise disturbances; therefore, in practice the selection of spanwise disturbance wavelengths has not been based on similar considerations as those used for selecting the streamwise wavelengths. This does not constitute a shortcoming of the stability analysis, but merely points to its perceived lack of utility in achieving the goals of threedimensional simulations. 


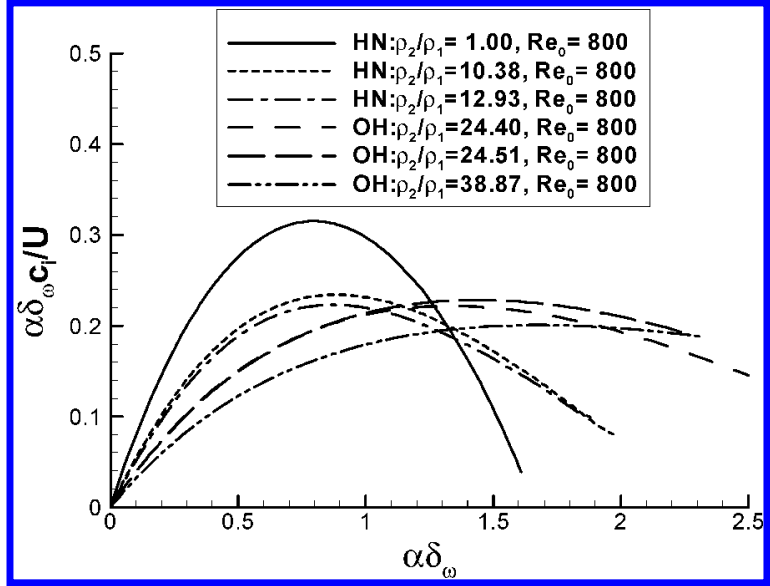

a)

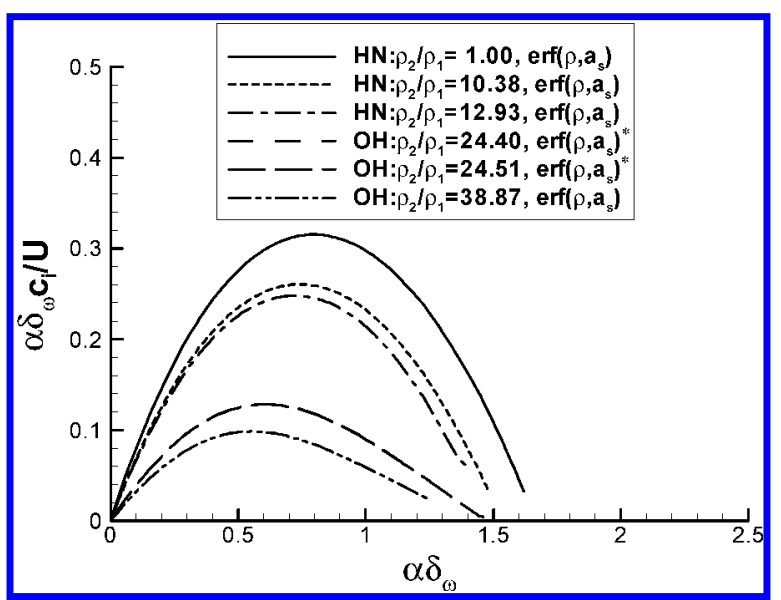

b)

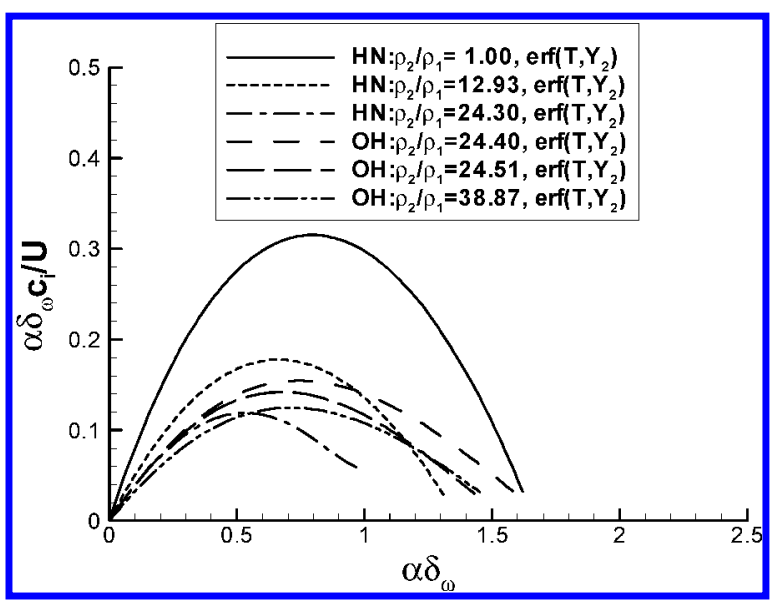

c)

Fig. 9 Stability curves showing the effect of species system and density stratification for a) similarity solution mean profile, b) $\operatorname{erf}\left(\rho, a_{s}\right)$, and c) $\operatorname{erf}\left(T, Y_{2}\right): \star$, overlapping curves.

\section{Conclusions}

A similarity analysis was conducted followed by a temporal stability analysis for conservation equations describing a temporal mixing layer at all pressures, including supercritical ones. One of the peculiarities of these conservation equations is that additional to the traditional Fick and Fourier transport coefficients, there is a new transport coefficient, the thermal diffusion factor, which couples molar and heat fluxes. This coupling occurs through the Soret term in the species equations and the Dufour term in the energy equation. To close the system, the conservation equations were here complemented by a real-gas EOS. Furthermore, we specified transport coefficients that depend on the thermodynamic quantities.

The similarity analysis was performed under the one-dimensional assumption. The velocity and mass fraction similarity solutions were shown to be close to the erf in the similarity variable, whereas the temperature did not follow the erf variation. For HN layers, the temperature adjacent to the colder (heptane) stream dropped below the freestream temperature. For $\mathrm{OH}$ layers, the temperature near the colder (oxygen) stream also dropped below the freestream temperature, but additionally the temperature next to the warmer (hydrogen) stream exceeded the freestream temperature. This temperature behavior was attributed to real-gas thermodynamics and to Schmidt and Prandtl number variations.

The two-dimensional temporal inviscid stability analysis was conducted using the similarity solution as the mean flow and also by adopting as an approximation an erf basic flow. The results showed that the unstable growth rates for the compressible flow are smaller at all wave numbers than the equivalent growth rates found from an incompressibleanalysis. Also, with increasingdensity stratification, the most unstable wavelengths occurred at smaller wave numbers. For given freestream conditions, the similarity profiles were more unstable and had shortermore unstable wavelengths than the erf profiles, as well as a wider range of unstable wave numbers. Constant density layers were found to have different stability characteristics than incompressible layers, indicating an EOS effect through the speed of sound.

For the three-dimensional temporal stability analysis, additional to the wavelength of the perturbation, the angle of the wave direction in the streamwise-spanwise plane must be specified; however, there is a maximum angle above which unstable wavelength perturbations cannot be found. For the purpose of applications to threedimensional flow simulations, this implies that the use of a shorter wavelength in the streamwise direction will be counterbalanced by the need to use a longer wavelength in the spanwise direction.

\section{Appendix A: Freestream Velocities}

The method to be described for determining the freestream velocities was proposed by Papamoschou and Roshko ${ }^{22}$ based on their experimental results for PG mixtures. The essence of the method is to use the isentropic relation

$$
\mathrm{d} h=\left(\frac{\partial h}{\partial p}\right)_{s} \mathrm{~d} p
$$

and the energy balance

$$
\mathrm{d} h=-\mathrm{d}\left[\frac{1}{2}\left(u_{1}-U_{c}\right)^{2}\right]
$$

to equate the static and stagnation pressures of the two streams. For computational purposes, in particular to avoid the need to calculate the entropy, Eq. (A1) is rewritten as

$$
\frac{\mathrm{d} h}{\mathrm{~d} p}=\left(\frac{\partial h}{\partial p}\right)_{T}+\left(\frac{\partial h}{\partial T}\right)_{p} \frac{\mathrm{d} T}{\mathrm{~d} p}, \quad \frac{\mathrm{d} T}{\mathrm{~d} p}=\left(\frac{\partial T}{\partial p}\right)_{s}
$$

where

$$
\begin{gathered}
\left(\frac{\partial h}{\partial p}\right)_{T}=\frac{1}{\rho}\left(1-\alpha_{v} T\right), \quad\left(\frac{\partial h}{\partial T}\right)_{p}=\frac{C_{p}}{m} \\
\left(\frac{\partial T}{\partial p}\right)_{s}=\frac{v \alpha_{v} T}{C_{p}}=\frac{\alpha_{v} T}{\rho C_{p} / m}
\end{gathered}
$$

A. PG Velocities

For a PG ( $\left.p=R_{u} T / v\right)$, Eqs. (A2) and (A3) become

$$
\frac{C_{p}}{m} \mathrm{~d} T=-\mathrm{d}\left[\frac{1}{2}\left(u_{1}-U_{c}\right)^{2}\right], \quad \frac{\mathrm{d} p}{p}=\frac{\gamma}{\gamma-1} \frac{\mathrm{d} T}{T}
$$


Table A1 Estimated convection velocity

\begin{tabular}{lcccccc}
\hline \hline Case & $\mathrm{HNa}$ & $\mathrm{HNb}$ & $\mathrm{HNc}$ & $\mathrm{OHa}$ & $\mathrm{OHb}$ & $\mathrm{OHc}$ \\
\hline$U_{1},{ }^{\mathrm{a}} \mathrm{m} / \mathrm{s}$ & 72.203 & 232.83 & 258.77 & 775.71 & 568.97 & 556.30 \\
$U_{2},{ }^{\mathrm{a}} \mathrm{m} / \mathrm{s}$ & -72.203 & -72.268 & -71.954 & -157.02 & -114.93 & -89.224 \\
$U_{c, \text { est }},{ }^{\mathrm{b}} \mathrm{m} / \mathrm{s}$ & 0 & 0 & 0 & 0 & 0 & 0 \\
$U_{c},{ }^{\mathrm{c}} \mathrm{m} / \mathrm{s}$ & 0 & 0 & 0.042 & 0 & 0.236 & 0.442 \\
$\left(U_{c}-U_{c, \text { est }}\right) /\left(U_{1}-U_{2}\right)$ & 0 & 0 & $1.3 \times 10^{-4}$ & 0 & $3.5 \times 10^{-4}$ & $6.8 \times 10^{-4}$ \\
\hline \hline
\end{tabular}

${ }^{\mathrm{a}}$ From Eq. (13). ${ }^{\mathrm{b}}$ From Eq. (A9). ${ }^{\mathrm{c}}$ From Eqs. (A2) and (A3).

where

$$
\gamma=C_{p} / C_{v}, \quad C_{v}=C_{p}-R_{u}
$$

(See Ref. 13 for the PG relations.) Assuming $C_{p}$ to be constant (implies $\gamma$ is constant) leads to

$$
\frac{T_{t}}{T}=1+\frac{1}{2} \frac{\left(u_{1}-U_{c}\right)^{2}}{\left(C_{p} / m\right) T}, \quad \frac{p_{t}}{p}=\left(\frac{T_{t}}{T}\right)^{\gamma /(\gamma-1)}
$$

where $T_{t}$ and $p_{t}$ are the stagnation temperature and pressure, respectively. Equating the static and stagnation pressures of the two streams

$$
\left[1+\frac{1}{2} \frac{\left(U_{1}-U_{c}\right)^{2}}{\left(C_{p_{1}} / m_{1}\right) T_{1}}\right]^{\gamma_{1} /\left(\gamma_{1}-1\right)}=\left[1+\frac{1}{2} \frac{\left(U_{2}-U_{c}\right)^{2}}{\left(C_{p_{2}} / m_{2}\right) T_{2}}\right]^{\gamma_{2} /\left(\gamma_{2}-1\right)}
$$

Supposing $\gamma_{1}=\gamma_{2}$ (implies $C_{p 1}=C_{p 2}$ ) leads to

$$
U_{c}=\frac{\sqrt{\rho_{1}} U_{1}+\sqrt{\rho_{2}} U_{2}}{\sqrt{\rho_{1}}+\sqrt{\rho_{2}}}
$$

which is the same as Eq. (7) of Papamoschou and Roshko.22 Then, for $U_{c}=0$, we obtain Eq. (13):

$$
U_{1}=\frac{2 M_{c} a_{s 1}}{\left[1+\left(a_{s 1} / a_{s 2}\right) \sqrt{\rho_{1} / \rho_{2}}\right]}, \quad U_{2}=-\sqrt{\frac{\rho_{1}}{\rho_{2}}} U_{1}
$$

Further manipulation of Eq. (A9) using the PG relation $a_{s}^{2}=\gamma p / \rho$ leads to Eq. (8) of Papamoschou and Roshko ${ }^{22}$; however, that equation restricts the convective Mach numbers of the two streams to be equal.

\section{B. Real Gas Velocities}

To assess the applicability of Eq. (A9) to real gases, Eqs. (A2) and (A3) are solved using real-gas EOS. A fourth-order Runge-Kutta scheme is used to integrate the system, which for the optimized value of $U_{c}$ will have the same stagnation pressure in both streams. The values of $U_{1}$ and $U_{2}$ are obtained from Eq. (13) with $M_{c}=0.4$ and determine the enthalpy change. The freestream (static) pressure and temperatures are specified and are the initial conditions for the integration of Eq. (A3). The final conditions are the stagnation pressure and temperatures, which satisfy the enthalpy change. The optimum $U_{c}$ is obtained using a bisection method. The results are summarized in Table A1. For the cases considered,Eq. (A9) leads to a convection velocity within $1 \%$ (relative to $\Delta U_{0}$ ) of the optimum $U_{c}$ and, therefore, is clearly an excellent approximation to the optimum $U_{c}$ for the RGNI mixtures considered.

\section{Appendix B: Analysis of Stability Equation}

To facilitate the analysis, Eq. (28) is rewritten as

$\frac{\mathrm{d}}{\mathrm{d} x_{2}}\left[\frac{1}{\bar{\rho}\left(\bar{u}_{1} \cos \phi-c\right)^{2}} \frac{\mathrm{d} \hat{p}}{\mathrm{~d} x_{2}}\right]-\left[\frac{1}{\bar{\rho}\left(\bar{u}_{1} \cos \phi-c\right)^{2}}-\frac{1}{\bar{\rho} a_{s}^{2}}\right] \alpha^{2} \hat{p}=0$
A. Solution for $\alpha=0$

For $\alpha=0$, Eq. (B1) becomes

$$
\begin{gathered}
\frac{\mathrm{d}}{\mathrm{d} x_{2}}\left[\frac{1}{\bar{\rho}\left(\bar{u}_{1} \cos \phi-c\right)^{2}} \frac{\mathrm{d} \hat{p}}{\mathrm{~d} x_{2}}\right]=0 \\
\Rightarrow \frac{1}{\bar{\rho}\left(\bar{u}_{1} \cos \phi-c\right)^{2}} \frac{\mathrm{d} \hat{p}}{\mathrm{~d} x_{2}}=A \\
\Rightarrow \hat{p}=\int A \bar{\rho}\left(\bar{u}_{1} \cos \phi-c\right)^{2} \mathrm{~d} x_{2}
\end{gathered}
$$

where $A$ is a constant of integration. When the boundary condition is applied,

$$
\hat{p}(\infty)=\int_{-\infty}^{\infty} \bar{\rho}\left(\bar{u}_{1} \cos \phi-c\right)^{2} \mathrm{~d} x_{2}=0
$$

The real and imaginary parts of this expression are

$$
\begin{gathered}
\int_{-\infty}^{\infty} \bar{\rho}\left[\left(\bar{u}_{1} \cos \phi-c_{r}\right)^{2}-c_{i}^{2}\right] \mathrm{d} x_{2}=0 \\
2 i c_{i} \int_{-\infty}^{\infty} \bar{\rho}\left(\bar{u}_{1} \cos \phi-c_{r}\right) \mathrm{d} x_{2}=0
\end{gathered}
$$

If $c_{i}=0$, a quadratic equation for $c_{r}$ is obtained from Eq. (B4),

$\int_{-\infty}^{\infty} \bar{\rho}\left(\bar{u}_{1} \cos \phi\right)^{2} \mathrm{~d} x_{2}-2 c_{r} \int_{-\infty}^{\infty} \bar{\rho} \bar{u}_{1} \cos \phi \mathrm{d} x_{2}+c_{r}^{2} \int_{-\infty}^{\infty} \bar{\rho} \mathrm{d} x_{2}=0$

When $c_{i} \neq 0$ is supposed, Eq. (B5) yields

$$
\begin{aligned}
\int_{-\infty}^{\infty} \bar{\rho}\left(\bar{u}_{1} \cos \phi-c_{r}\right) \mathrm{d} x_{2} & =0 \\
\Rightarrow \int_{-\infty}^{\infty} \bar{\rho} \bar{u}_{1} \cos \phi \mathrm{d} x_{2} & =c_{r} \int_{-\infty}^{\infty} \bar{\rho} \mathrm{d} x_{2}
\end{aligned}
$$

When this result is substituted into Eq. (B4),

$$
\int_{-\infty}^{\infty} \bar{\rho}\left(\bar{u}_{1} \cos \phi\right)^{2} \mathrm{~d} x_{2}=\left(c_{r}^{2}+c_{i}^{2}\right) \int_{-\infty}^{\infty} \bar{\rho} \mathrm{d} x_{2}
$$

The solution for $c$ is

$$
c_{r}=\frac{\int_{-\infty}^{\infty} \bar{\rho} \bar{u}_{1} \cos \phi \mathrm{d} x_{2}}{\int_{-\infty}^{\infty} \bar{\rho} \mathrm{d} x_{2}}, \quad c_{r}^{2}+c_{i}^{2}=\frac{\int_{-\infty}^{\infty} \bar{\rho}\left(\bar{u}_{1} \cos \phi\right)^{2} \mathrm{~d} x_{2}}{\int_{-\infty}^{\infty} \bar{\rho} \mathrm{d} x_{2}}
$$

where for instability the positive root of $c_{i}$ is desired.

\section{B. Range of $c_{r}$}

Multiplying Eq. (B1) by $\hat{p}^{*}$, the complex conjugate of $\hat{p}$, and integrating

$$
\begin{aligned}
& \int_{-\infty}^{\infty} \hat{p}^{*} \frac{\mathrm{d}}{\mathrm{d} x_{2}}\left[\frac{1}{\bar{\rho}\left(\bar{u}_{1} \cos \phi-c\right)^{2}} \frac{\mathrm{d} \hat{p}}{\mathrm{~d} x_{2}}\right] \mathrm{d} x_{2} \\
& \quad-\int_{-\infty}^{\infty}\left[\frac{1}{\bar{\rho}\left(\bar{u}_{1} \cos \phi-c\right)^{2}}-\frac{1}{\bar{\rho} a_{s}^{2}}\right] \alpha^{2}|\hat{p}|^{2} \mathrm{~d} x_{2}=0
\end{aligned}
$$


Integrating the first term by parts,

$$
\begin{gathered}
\int_{-\infty}^{\infty} \hat{p}^{*} \frac{\mathrm{d}}{\mathrm{d} x_{2}}\left[\frac{1}{\bar{\rho}\left(\bar{u}_{1} \cos \phi-c\right)^{2}} \frac{\mathrm{d} \hat{p}}{\mathrm{~d} x_{2}}\right] \mathrm{d} x_{2} \\
=\left[\hat{p}^{*} \frac{1}{\bar{\rho}\left(\bar{u}_{1} \cos \phi-c\right)^{2}} \frac{\mathrm{d} \hat{p}}{\mathrm{~d} x_{2}}\right]_{-\infty}^{\infty} \\
-\int_{-\infty}^{\infty} \frac{1}{\bar{\rho}\left(\bar{u}_{1} \cos \phi-c\right)^{2}} \frac{\mathrm{d} \hat{p}}{\mathrm{~d} x_{2}} \frac{\mathrm{d} \hat{p}^{*}}{\mathrm{~d} x_{2}} \mathrm{~d} x_{2}
\end{gathered}
$$

Applying the boundary conditions $\hat{p}^{*}( \pm \infty)=0$ and substituting into Eq. (B10):

$\int_{-\infty}^{\infty}\left[\frac{1}{\bar{\rho}\left(\bar{u}_{1} \cos \phi-c\right)^{2}}\left(\left|\frac{\mathrm{d} \hat{p}}{\mathrm{~d} x_{2}}\right|^{2}+\alpha^{2}|\hat{p}|^{2}\right)-\frac{1}{\bar{\rho} a_{s}^{2}} \alpha^{2}|\hat{p}|^{2}\right] \mathrm{d} x_{2}=0$

The imaginary part of this expression is

$$
\begin{gathered}
2 c_{i} \int_{-\infty}^{\infty}\left[\left(\bar{u}_{1} \cos \phi-c_{r}\right) \frac{1}{\left|\left(\bar{u}_{1} \cos \phi-c\right)^{2}\right|^{2}} \frac{1}{\bar{\rho}}\right. \\
\left.\quad \times\left(\left|\frac{\mathrm{d} \hat{p}}{\mathrm{~d} x_{2}}\right|^{2}+\alpha^{2}|\hat{p}|^{2}\right)\right] \mathrm{d} x_{2}=0
\end{gathered}
$$

Therefore $c_{i}=0$, or because $\left(\bar{u}_{1} \cos \phi-c_{r}\right)$ must change sign at least once, $c_{r}$ lies in the range of $\bar{u}_{1} \cos \phi$. There is a singularity in Eq. (28) where $\bar{u}_{1} \cos \phi-c=0$.

\section{Acknowledgments}

This study was conducted at the Jet Propulsion Laboratory (JPL), California Institute of Technology, and sponsored jointly by the Air Force Office of Scientific Research under the direction of Julian Tishkoff, by the Army Research Office under the direction of David Mann under an interagency agreement with NASA, and by NASA Marshall Space Flight Center, under the direction of John Hutt. The computationalresources were provided by the JPL Supercomputing Center.

\section{References}

${ }^{1}$ Prausnitz, J., Lichtenthaler, R., and de Azevedo, E., Molecular Thermodynamics for Fluid-Phase Equilibrium, Prentice-Hall, Upper Saddle River, NJ, 1986.

${ }^{2}$ Hirshfelder, J., Curtis, C., and Bird, R., Molecular Theory of Gases and Liquids, Wiley, New York, 1964.

${ }^{3}$ Clifford, T., Fundamentals of Supercritical Fluids, Oxford Univ. Press, Oxford, 1999.

${ }^{4}$ Moser, R., and Rogers, M., "Mixing Transition and the Cascade to Small Scales in a Plane Mixing Layer," Physics of Fluids A, Vol. 3, No. 5, 1991, pp. 1128-1134.
${ }^{5}$ Metcalfe, R., Orszag, S., Brachet, M., Menon, S., and Riley, J., "Secondary Instability of a Temporally Growing Mixing Layer," Journal of Fluid Mechanics, Vol. 184, 1987, pp. 207-243.

${ }^{6}$ Drazin, P., and Reid, W., Hydrodynamic Stability, Cambridge Univ. Press, Cambridge, England, U.K., 1981.

${ }^{7}$ Jackson, T., and Grosch, C., "Inviscid Spatial Stability of a Compressible Mixing Layer. 2. The Flame Sheet Model," Journal of Fluid Mechanics, Vol. 217, 1990, pp. 391-420.

- ${ }^{8}$ Jackson, T., and Grosch, C., "Inviscid Spatial Stability of a Compressible Mixing Layer. 3. Effect of Thermodynamics," Journal of Fluid Mechanics, Vol. 224, 1991, pp. 159-175.

${ }^{9}$ Shin, D., and Ferziger, J., "Linear Stability of the Reacting Mixing Layer," AIAA Journal, Vol. 29, No. 10, 1991, pp. 1634-1642.

${ }^{10}$ Lu, G., and Lele, S., "On the Density Ratio Effect on the Growth Rate of a Compressible Mixing Layer," Physics of Fluids, Vol. 6, No. 2, 1994, pp. 1073-1075

${ }^{11}$ Kozusko, F., Lasseigne, D., Grosch, C., and Jackson, T., "The Stability of Compressible Mixing Layers in Binary Gases," Physics of Fluids, Vol. 8, No. 7, 1996, pp. 1954-1963.

${ }^{12}$ Miller, R., Harstad, K., and Bellan, J., "Direct Numerical Simulations of Supercritical Fluid Mixing Layers Applied to Heptane-Nitrogen," Journal of Fluid Mechanics, Vol. 436, 2001, pp. 1-39.

13 Okong'o, N., and Bellan, J., "Consistent Boundary Conditions for Multicomponent Real Gas Mixtures Based on Characteristic Waves," Journal of Computational Physics, Vol. 176, 2002, pp. 330-344.

$>14$ Okong'o, N., and Bellan, J., "Direct Numerical Simulation of a Transitional Supercritical Binary Mixing Layer: Heptane and Nitrogen," Journal of Fluid Mechanics, Vol. 464, 2002, pp. 1-34

${ }^{15}$ Okong'o, N., Harstad, K., and Bellan, J., "Direct Numerical Simulations of $\mathrm{O}_{2} / \mathrm{H}_{2}$ Temporal Mixing Layers Under Supercritical Conditions," AIAA Journal, Vol. 40, No. 5, 2002, pp. 914-926.

$>{ }^{16}$ Keizer, J., Statistical Thermodynamics of Nonequilibrium Processes, Springer-Verlag, New York, 1987.

${ }^{17}$ Sarman, S., and Evans, D., "Heat Flux and Mass Diffusion in Binary Lennard-Jones Mixtures," Physics Review A, Vol. 45, No. 4, 1992 pp. $2370-2379$

${ }^{18}$ Harstad, K., and Bellan, J., "An All-Pressure Fluid-Drop Model Applied to a Binary Mixture: Heptane in Nitrogen," International Journal of Multiphase Flow, Vol. 26, No. 10, 2000, pp. 1675-1706.

${ }^{19}$ Harstad, K., Miller, R., and Bellan, J., "Efficient High Pressure State Equations," AICHE Journal, Vol. 43, No. 6, 1997, pp. 1605-1610.

${ }^{20}$ Harstad, K., and Bellan, J., "Isolated Fluid Oxygen Drop Behavior in Fluid Hydrogen at Rocket Chamber Pressures," International Journal of Heat and Mass Transfer, Vol. 41, 1998, pp. 3537-3550.

${ }^{21}$ Harstad, K., and Bellan, J., "The $D^{2}$ Variation For Isolated LOX Drops and Polydisperse Clusters in Hydrogen at High Temperature and Pressures," Combustion and Flame, Vol. 124, 2001, pp. 535-550.

${ }^{22}$ Papamoschou, D., and Roshko, A., "The Compressible Turbulent Shear Layer: An Experimental Study,' Journal of Fluid Mechanics, Vol. 197, 1988, pp. 453-477.

${ }^{23}$ Brown, G., and Roshko, A., "On Density Effects and Large Structure in Turbulent Mixing Layers," Journal of Fluid Mechanics, Vol. 64, No. 4, 1974, pp. 775-816.

${ }^{24}$ Harstad, K., and Bellan, J., "The Lewis Number Under Supercritical Conditions," InternationalJournal of Heat andMass Transfer, Vol. 42, 1999 pp. 961-970.

W. Dahm Associate Editor 


\section{This article has been cited by:}

1. Pasquale Eduardo Lapenna, Francesco Creta. 2017. Mixing under transcritical conditions: An a-priori study using direct numerical simulation. The Journal of Supercritical Fluids 128, 263-278. [Crossref]

2. Martin J. Seidl, Manfred Aigner, Roman Keller, Peter Gerlinger. 2017. CFD simulations of turbulent nonreacting and reacting flows for rocket engine applications. The Journal of Supercritical Fluids 121, 63-77. [Crossref]

3. Peter C. Ma, Daniel Banuti, Jean-Pierre Hickey, Matthias Ihme. Numerical framework for transcritical real-fluid reacting flow simulations using the flamelet progress variable approach . [Citation] [PDF] [PDF Plus]

4. Chowdhury Ashraf, Abhishek Jain, Yuan Xuan, Adri C. T. van Duin. 2017. ReaxFF based molecular dynamics simulations of ignition front propagation in hydrocarbon/oxygen mixtures under high temperature and pressure conditions. Phys. Chem. Chem. Phys. 19:7, 5004-5017. [Crossref]

5. Qing-fei Fu, Li-zi Qin, Li-jun Yang. 2017. Spatial-Temporal Instability of an Inviscid Shear Layer. International Journal of Aerospace Engineering 2017, 1-7. [Crossref]

6. Qing-Fei Fu, Li-Jun Yang. 2016. Temporal Instability of a Transcritical Shear Layer. Journal of Propulsion and Power 32:5, 1292-1297. [Citation] [Full Text] [PDF] [PDF Plus]

7. Daniel T. Banuti, Volker Hannemann, Klaus Hannemann, Bernhard Weigand. 2016. An efficient multi-fluid-mixing model for real gas reacting flows in liquid propellant rocket engines. Combustion and Flame 168, 98-112. [Crossref]

8. Lu Qiu, Rolf D. Reitz. 2015. An investigation of thermodynamic states during high-pressure fuel injection using equilibrium thermodynamics. International Journal of Multiphase Flow 72, 24-38. [Crossref]

9. Lu Qiu, Rolf D. Reitz. 2014. Simulation of supercritical fuel injection with condensation. International Journal of Heat and Mass Transfer 79, 1070-1086. [Crossref]

10. Daniel T. Banuti, Klaus Hannemann. Supercritical Pseudo-Boiling and its Relevance for Transcritical Injection . [Citation] [PDF] [PDF Plus]

11. F. Battista, F. Picano, C. M. Casciola. 2014. Turbulent mixing of a slightly supercritical van der Waals fluid at lowMach number. Physics of Fluids 26:5, 055101. [Crossref]

12. Jean-Pierre Hickey, Matthias Ihme. Large Eddy Simulation of Supercritical Mixing and Combustion for Rocket Applications . [Citation] [PDF] [PDF Plus]

13. Hiroumi Tani, Susumu Teramoto, Nobuhiro Yamanishi, Koji Okamoto. 2013. A numerical study on a temporal mixing layer under transcritical conditions. Computers \& Fluids 85, 93-104. [Crossref]

14. Jean-Pierre Hickey, Peter C. Ma, Matthias Ihme, Siddharth S. Thakur. Large Eddy Simulation of Shear Coaxial Rocket Injector: Real Fluid Effects . [Citation] [PDF] [PDF Plus]

15. Enrica Masi, Josette Bellan, Kenneth Harstad. Direct Numerical Simulation of High-Pressure Multispecies Turbulent Mixing in the Cold Ignition Regime . [Citation] [PDF] [PDF Plus]

16. Nora Okong'o, Josette Bellan. 2010. Small-scale dissipation in binary-species, thermodynamically supercritical, transitional mixing layers. Computers \& Fluids 39:7, 1112-1124. [Crossref]

17. EZGI S. TASKINOGLU, JOSETTE BELLAN. 2010. A posteriori study using a DNS database describing fluid disintegration and binary-species mixing under supercritical pressure: heptane and nitrogen. Journal of Fluid Mechanics 645, 211. [Crossref]

18. Ezgi Taskinoglu, Josette Bellan. An a Posteriori Study of a DNS Database Describing Supercritical Binary-Species Mixing . [Citation] [PDF] [PDF Plus]

19. Josette Bellan, Nora Okong'o. Small-Scale Dissipation in Supercritical, Transitional Mixing Layers . [Citation] [PDF] [PDF Plus]

20. Laurent Selle, Josette Bellan, Kenneth Harstad. Modeling of the Energy Equation for LES of Flows at Supercritical Pressure . [Citation] [PDF] [PDF Plus]

21. LAURENT C. SELLE, NORA A. OKONG'O, JOSETTE BELLAN, KENNETH G. HARSTAD. 2007. Modelling of subgrid-scale phenomena in supercritical transitional mixing layers: an a priori study. Journal of Fluid Mechanics 593. . [Crossref]

22. Laurent Selle, Josette Bellan, Kenneth Harstad. Novel Subgrid Modeling of the LES Equations Under Supercritical Pressure . [Citation] [PDF] [PDF Plus]

23. JOSETTE BELLAN. 2006. THEORY, MODELING AND ANALYSIS OF TURBULENT SUPERCRITICAL MIXING. Combustion Science and Technology 178:1-3, 253-281. [Crossref] 
24. S. CANDEL*, M. JUNIPER $\dagger$, G. SINGLA, P. SCOUFLAIRE, C. ROLON. 2006. STRUCTURE AND DYNAMICS OF CRYOGENIC FLAMES AT SUPERCRITICAL PRESSURE. Combustion Science and Tecbnology 178:1-3, 161-192. [Crossref]

25. N. Okong'o, Josette Bellan. A Priori Analysis of Subgrid-Scale Models for Large Eddy Simulations of Supercritical Binary-Species Mixing Layers . [Citation] [PDF] [PDF Plus]

26. Nora Okong'o, Josette Bellan. 2004. Perturbation and initial Reynolds number effects on transition attainment of supercritical, binary, temporal mixing layers. Computers \& Fluids 33:8, 1023-1046. [Crossref]

27. N. Okong'o, J. Bellan. 2004. Turbulence and fluid-front area production in binary-species, supercritical, transitional mixing layers. Physics of Fluids 16:5, 1467-1492. [Crossref] 